\title{
MATING THE BASILICA WITH A SIEGEL DISK
}

\author{
JONGUK YANG
}

Abstract. Let $f_{\mathbf{S}}$ be a quadratic polynomial with a fixed Siegel disc of bounded type. Using an adaptation of complex a priori bounds for critical circle maps, we prove that $f_{\mathbf{S}}$ is conformally mateable with the basilica polynomial $f_{\mathbf{B}}(z):=z^{2}-1$.

\section{The Definition of MAting}

The simplest non-linear examples of holomorphic dynamical systems are given by quadratic polynomials in $\mathbb{C}$. By a linear change of coordinates, any quadratic polynomial can be uniquely normalized as

$$
f_{c}(z):=z^{2}+c \quad \text { for some } \quad c \in \mathbb{C} .
$$

This is referred to as the quadratic family.

The critical points for $f_{c}$ are $\infty$ and 0 . Observe that $\infty$ is a superattracting fixed point for $f_{c}$. Let $\mathbf{A}_{c}^{\infty}$ be the attracting basin of $\infty$. It follows from the maximum modulus principle that $\mathbf{A}_{c}^{\infty}$ is a connected set. The complement of $\mathbf{A}_{c}^{\infty}$ is called the filled Julia set $K_{c}$. The boundary of $K_{c}$ is equal to the Julia set $J_{c}$.

The non-escape locus in the parameter space for $f_{c}$ (referred to as the Mandelbrot set) is defined as a compact subset of $\mathbb{C}$ :

$$
\mathcal{M}:=\left\{c \in \mathbb{C} \mid 0 \notin \mathbf{A}_{c}^{\infty}\right\},
$$

which is known to be connected (see $[\mathrm{DH}]$ ). It is not difficult to prove that $J_{c}$ is connected (and therefore, $\mathbf{A}_{c}^{\infty}$ is simply connected) if and only if $c \in \mathcal{M}$. In fact, if $c \notin \mathcal{M}$, then $J_{c}=K_{c}$ is a Cantor set, and the dynamics of $f_{c}$ restricted to $J_{c}$ is conjugate to the dyadic shift map (see [M2]). We also define the following subset of the Mandelbrot set:

$$
\mathcal{L}:=\left\{c \in \mathcal{M} \mid J_{c} \text { is locally connected }\right\} .
$$

The quadratic family has been the center of attention in holomorphic dynamics for the past three decades, and we now have an almost complete understanding of its dynamics (see e.g. [M2]). It should be noted, however, that the main conjecture in the field (the local connectedness property of the Mandelbrot set, or $M L C$ for short) remains open (see $[\mathrm{DH}]$ ).

In contrast to the quadratic family, the dynamics of non-polynomial quadratic rational maps is still a wide open area of research. In this section, we describe a construction originally put forward by Douady and Hubbard (see [Do] which

Received by the editors November 20, 2014 and, in revised form, June 3, 2015, July 26, 2015, and September 10, 2015.

2010 Mathematics Subject Classification. Primary 37F10, 37F45, 37F50; Secondary 37F25, $37 \mathrm{~F} 30$. 


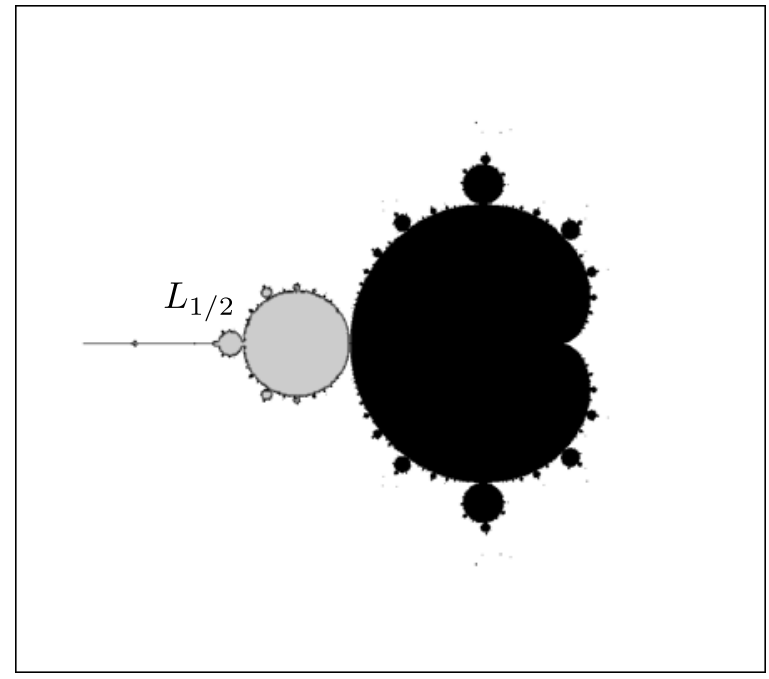

Figure 1. The Mandelbrot set $\mathcal{M}$. The $1 / 2$-limb $L_{1 / 2}$ is highlighted.

produces quadratic rational maps by combining the dynamics of two quadratic polynomials. Let

Suppose $c \in \mathcal{L}$. Since $J_{c}$ is connected, $\mathbf{A}_{c}^{\infty}$ must be a simply connected domain.

$$
\phi_{c}: \mathbf{A}_{c}^{\infty} \rightarrow \mathbb{D}
$$

be the unique conformal Riemann mapping such that $\phi_{c}(\infty)=0$ and $\phi_{c}^{\prime}(\infty)>0$. It is not difficult to prove that the following diagram commutes:

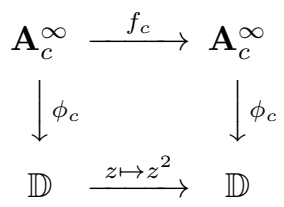

and hence, $\phi_{c}$ is the Böttcher uniformization of $f_{c}$ on $\mathbf{A}_{c}^{\infty}$. Moreover, since $J_{c}$ is locally connected, Carathéodory's theory implies that the inverse of $\phi_{c}$ extends continuously to the boundary of $\mathbb{D}$ (see [M1]). If we let

$$
\tau_{c}:=\left.\phi_{c}^{-1}\right|_{\partial \mathbb{D}},
$$

we obtain a continuous parametrization of $J_{c}$ by the unit circle $\partial \mathbb{D}=\mathbb{R} / \mathbb{Z}$ known as a Carathéodory loop. Observe that $f_{c}$, when restricted to $J_{c}$, acts via $\tau_{c}$ as the angle doubling map:

$$
f_{c}\left(\tau_{c}(t)\right)=\tau_{c}(2 t)
$$

Now, suppose $c_{1}, c_{2} \in \mathcal{L}$. Using $\tau_{c_{1}}$ and $\tau_{c_{2}}$, we can glue the dynamics of $f_{c_{1}}$ and $f_{c_{2}}$ together to construct a new dynamical system as follows.

First we construct a new dynamical space $K_{c_{1}} \vee K_{c_{2}}$ by gluing the filled Julia sets $K_{c_{1}}$ and $K_{c_{2}}$ :

$$
K_{c_{1}} \vee K_{c_{2}}:=\left(K_{c_{1}} \sqcup K_{c_{2}}\right) /\left\{\tau_{c_{1}}(t) \sim \tau_{c_{2}}(-t)\right\}
$$


We refer to the resulting equivalence relation $\sim$ as ray equivalence, and denote it by $\sim_{\text {ray }}$. For a point $x$ in $K_{c_{1}}$ or $K_{c_{2}}$, we denote the ray equivalency class of $x$ by $[x]_{\text {ray }}$.

We now define a new map

$$
f_{c_{1}} \vee f_{c_{2}}: K_{c_{1}} \vee K_{c_{2}} \rightarrow K_{c_{1}} \vee K_{c_{2}}
$$

called the formal mating of $f_{c_{1}}$ and $f_{c_{2}}$, by letting $f_{c_{1}} \vee f_{c_{2}} \equiv f_{c_{1}}$ on $K_{c_{1}}$ and $f_{c_{1}} \vee f_{c_{2}} \equiv f_{c_{2}}$ on $K_{c_{2}}$.

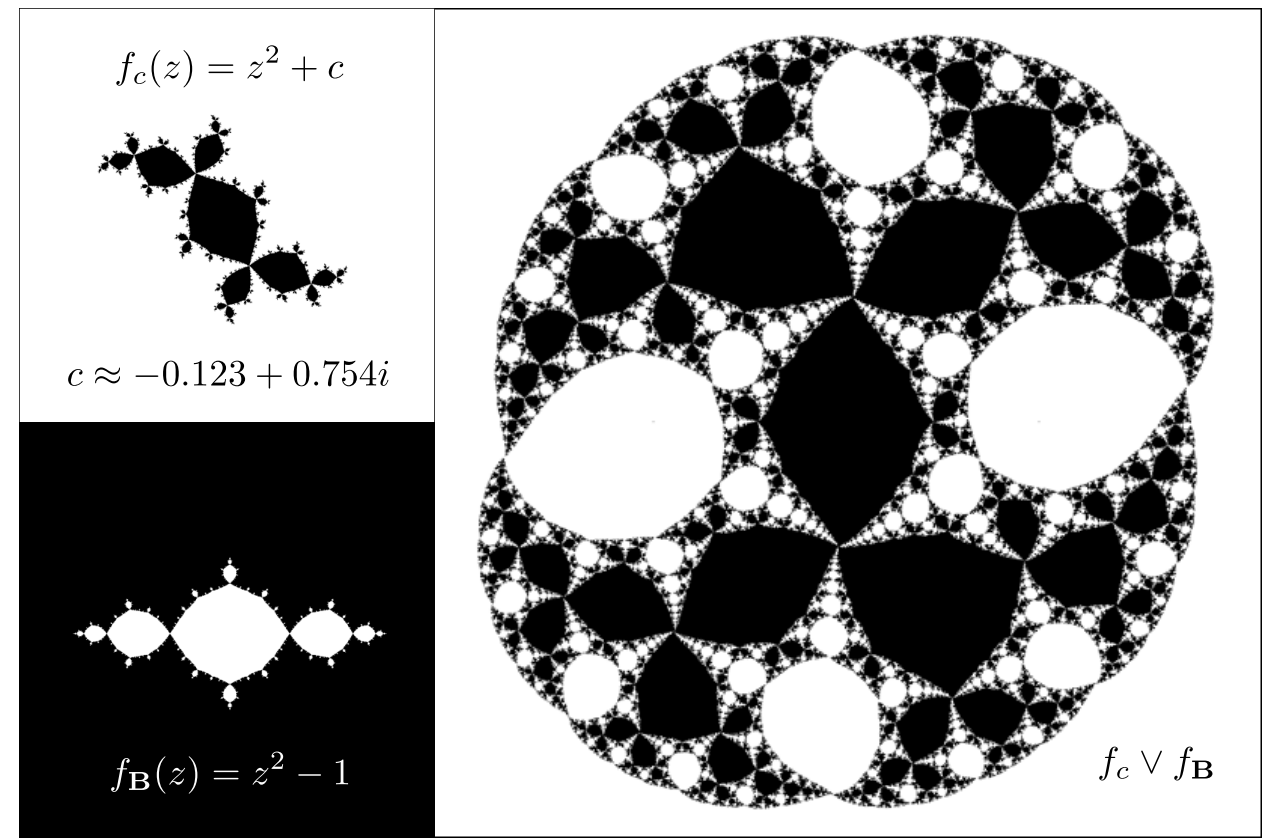

Figure 2. The Douady rabbit $f_{c}$ with $c \approx-0.123+0.754 i$ mated with the basilica polynomial $f_{\mathbf{B}}$.

If the space $K_{c_{1}} \vee K_{c_{2}}$ is homeomorphic to the 2-sphere, then $f_{c_{1}}$ and $f_{c_{2}}$ are said to be topologically mateable. If, in addition, there exists a quadratic rational map $R$ and a homeomorphism

$$
\Lambda: K_{c_{1}} \vee K_{c_{2}} \rightarrow \hat{\mathbb{C}}
$$

such that $\Lambda$ is conformal on $\stackrel{\circ}{K}_{c_{1}} \sqcup \stackrel{\circ}{K}_{c_{2}} \subset K_{c_{1}} \vee K_{c_{2}}$, and the following diagram commutes:

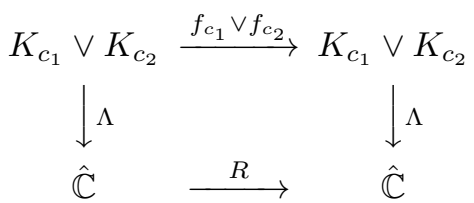

then $f_{c_{1}}$ and $f_{c_{2}}$ are said to be conformally mateable. The quadratic rational map $R$ is called a conformal mating of $f_{c_{1}}$ and $f_{c_{2}}$. We also say that $R$ realizes the conformal mating of $f_{c_{1}}$ and $f_{c_{2}}$.

In applications, it is sometimes more useful to work with the following reformulation of the definition of conformal mateability: 
Proposition 1.1. Suppose $c_{1}, c_{2} \in \mathcal{L}$. Then $f_{c_{1}}$ and $f_{c_{2}}$ are conformally mateable if and only if there exists a pair of continuous maps

$$
\Lambda_{1}: K_{c_{1}} \rightarrow \hat{\mathbb{C}} \quad \text { and } \quad \Lambda_{2}: K_{c_{2}} \rightarrow \hat{\mathbb{C}}
$$

such that for all $i, j \in\{1,2\}$ the following three conditions are satisfied:

(i) $\Lambda_{i}(z)=\Lambda_{j}(w)$ if and only if $z \sim_{\text {ray }} w$,

(ii) $\Lambda_{i}$ is conformal on $\stackrel{\circ}{K}_{c_{i}}$, and

(iii) there exists a rational function $R$ of degree 2 such that the following diagrams commute:

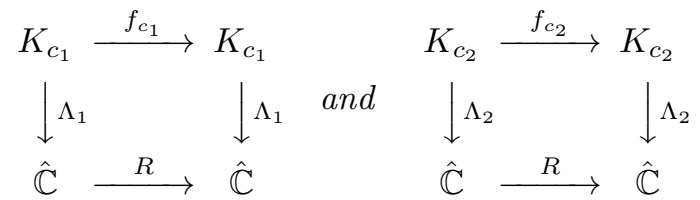

Corollary 1.2. Suppose $R$ is a conformal mating of $f_{c_{1}}$ and $f_{c_{2}}$ for some $c_{1}, c_{2} \in \mathcal{L}$. Then $R$ has a locally connected Julia set $J(R)$.

Proof. Let $\Lambda_{1}: K_{c_{1}} \rightarrow \hat{\mathbb{C}}$ and $\Lambda_{2}: K_{c_{2}} \rightarrow \hat{\mathbb{C}}$ be as given in Proposition 1.1. Note that

$$
J(R)=\Lambda_{1}\left(J_{c_{1}}\right)=\Lambda_{2}\left(J_{c_{2}}\right) .
$$

Since the continuous image of a compact locally connected set is locally connected, the result follows.

Example 1.3. For $c \in \mathcal{L}$, the quadratic polynomial $f_{c}$ is trivially conformally mateable with the squaring map $f_{0}(z)=z^{2}$. This follows from choosing $\Lambda_{1}$ and $\Lambda_{2}$ in Proposition 1.1 to be the identity map on $K_{c}$ and the inverse of the Böttcher uniformization of $f_{c}$ on $\mathbf{A}_{c}^{\infty}$ respectively. Note that the conformal mating of $f_{c}$ and $f_{0}$ is realized by $f_{c}$ itself. The following result shows that with the exception of this trivial case, the mating construction always yields a non-polynomial dynamical system.

Proposition 1.4. Suppose a quadratic polynomial $P: \mathbb{C} \rightarrow \mathbb{C}$ is a conformal mating of $f_{c_{1}}$ and $f_{c_{2}}$ for some $c_{1}, c_{2} \in \mathcal{L}$. Then either $f_{c_{1}}$ or $f_{c_{2}}$ must be equal to the squaring map $f_{0}$.

Proof. Let $J(P)$ and $\mathbf{A}_{P}^{\infty}$ denote the Julia set and the attracting basin of infinity for $P$ respectively. We have

$$
J(P)=\Lambda_{1}\left(J_{c_{1}}\right)=\Lambda_{2}\left(J_{c_{2}}\right) .
$$

Hence, $\mathbf{A}_{P}^{\infty}$ must be contained in either $\Lambda_{1}\left(\stackrel{\circ}{K}_{c_{1}}\right)$ or $\Lambda_{2}\left(\stackrel{\circ}{K}_{c_{2}}\right)$. Assume for concreteness that it is contained in the former. Since $\left.\Lambda_{1}\right|_{K_{K_{1}}}$ is conformal, and

$$
f_{c_{1}}(z)=\Lambda_{1}^{-1} \circ P \circ \Lambda_{1}(z) \quad \text { for all } \quad z \in K_{c_{1}},
$$

we see that $\Lambda_{1}^{-1}(\infty)$ must be a superattracting fixed point for $f_{c_{1}}$. The only member in the quadratic family that has a bounded superattracting fixed point is the squaring map $f_{0}$.

Example 1.5. Consider the formal mating of the basilica polynomial $f_{\mathbf{B}}(z):=$ $f_{-1}(z)=z^{2}-1$ with itself. The glued space $K_{\mathbf{B}} \vee K_{\mathbf{B}}$ consists of infinitely many spheres connected together at discrete nodal points (refer to Section 5.1 for the structural properties of $K_{\mathbf{B}}$ ). Hence, it is not homeomorphic to the 2-sphere. 
Therefore, $f_{\mathbf{B}}$ is not conformally mateable with itself (since it is not even topologically mateable with itself). This is actually a specific instance of a more general result, which we state below.

Let $H_{0}$ be the principal hyperbolic component defined as the set of $c \in \mathcal{M}$ for which $f_{c}$ has an attracting fixed point $z_{c} \in \mathbb{C}$. It is conformally parametrized by the multiplier of $z_{c}$ :

$$
\lambda: c \mapsto f_{c}^{\prime}\left(z_{c}\right)
$$

(see e.g. [M2 ). Note that $\lambda$ extends to a homeomorphism between $\overline{H_{0}}$ and $\overline{\mathbb{D}}$.

A connected component of $\mathcal{M} \backslash \overline{H_{0}}$ is called a limb. It is known (see e.g. [M2]) that the closure of every limb intersects $\partial H_{0}$ at a single point. Moreover, the image of this point under $\lambda$ is a root of unity. Henceforth, the limb growing from the point $\lambda^{-1}\left(e^{2 \pi i p / q}\right)$ for some $p / q \in \mathbb{Q}$ will be denoted by $L_{p / q}$. For example, the parameter value -1 for the basilica polynomial $f_{\mathbf{B}}(z)=z^{2}-1$ is contained in the $1 / 2$-limb $L_{1 / 2}$.

The following standard observation is due to Douady [Do]:

Proposition 1.6. Suppose $c_{1}$ and $c_{2}$ are contained in complex conjugate limbs $L_{p / q}$ and $L_{-p / q}$ of the Mandelbrot set $\mathcal{M}$. Then $f_{c_{1}}$ and $f_{c_{2}}$ are not topologically mateable.

Proof. There exists a unique repelling fixed point $\alpha_{1} \in K_{1}$ (resp. $\alpha_{2} \in K_{2}$ ) such that $K_{1} \backslash\left\{\alpha_{1}\right\}$ (resp. $K_{2} \backslash\left\{\alpha_{2}\right\}$ ) is disconnected. Since $c_{1}$ and $c_{2}$ are contained in complex conjugate limbs, $\alpha_{1}$ and $\alpha_{2}$ are in the same ray equivalency class. Hence they are glued together to a single point in $K_{c_{1}} \vee K_{c_{2}}$. Removing this single point from $K_{c_{1}} \vee K_{c_{2}}$ leaves it disconnected, which is impossible if $K_{c_{1}} \vee K_{c_{2}}$ is homeomorphic to the 2-sphere. For more details, see [M2].

\section{Matings With the Basilica POLYNOMial}

Matings can be particularly useful in describing the dynamics in certain oneparameter families of rational maps. The best studied example of such a family is

$$
R_{a}(z):=\frac{a}{z^{2}+2 z} \quad \text { for } \quad a \in \mathbb{C} \backslash\{0\},
$$

which is referred to as the basilica family.

The critical points for $R_{a}$ are $\infty$ and -1 . Observe that $\{\infty, 0\}$ is a superattracting 2-periodic orbit for $R_{a}$. Let $\mathcal{A}_{a}^{\infty}$ be the attracting basin of $\{\infty, 0\}$. The boundary of $\mathcal{A}_{a}^{\infty}$ is equal to the Julia set $J\left(R_{a}\right)$.

Proposition 2.1. Suppose $f: \hat{\mathbb{C}} \rightarrow \hat{\mathbb{C}}$ is a quadratic rational map with a superattracting 2-periodic orbit. Then by a linear change of coordinates, $f$ can be normalized as either:

(i) $R_{a}$ for some $a \in \mathbb{C} \backslash\{0\}$, or

(ii) $z \mapsto \frac{1}{z^{2}}$.

Proof. By a linear change of coordinates, we may assume that $f$ has a superattracting 2-periodic orbit $\{\infty, 0\}$ with a critical point at $\infty$. Let

$$
f(z)=\frac{a_{2} z^{2}+a_{1} z+a_{0}}{b_{2} z^{2}+b_{1} z+b_{0}} .
$$


Since $f(\infty)=0$ and $f(0)=\infty$, we have $a_{2}=b_{0}=0, b_{2} \neq 0$ and $a_{0} \neq 0$. If $a_{1} \neq 0$, then for $r$ sufficiently large, we have

$$
f\left(r e^{\theta}\right) \sim \frac{a_{1}}{b_{2} r} e^{-\theta} .
$$

This implies that $\infty$ cannot be a critical point for $f$ by the argument principle. Hence, we must have $a_{1}=0$. These observations yield the following expression for $f:$

$$
f(z)=\frac{a}{z^{2}+b z} \quad \text { with } \quad a \in \mathbb{C} \backslash\{0\} .
$$

If the second critical point for $f$ is equal to 0 , then by using a similar argument as above, we see that $b=0$. In this case, we have

$$
f(\lambda z) / \lambda=\frac{1}{z^{2}}
$$

where $\lambda$ is a cube root of $a$.

On the other hand, if the second critical point for $f$ is not equal to 0 , then we may assume by a linear change of coordinates that it is equal to -1 . A straightforward computation shows that $f^{\prime}(-1)=0$ if and only if $b=2$, which means $f=R_{a}$ as claimed.

Analogously to $\mathcal{M}$, the non-escape locus in the parameter space for $R_{a}$ is defined as

$$
\mathcal{M}_{\mathbf{B}}:=\left\{a \in \mathbb{C} \backslash\{0\} \mid-1 \notin \mathcal{A}_{a}^{\infty}\right\} .
$$

We also define the following subset of $\mathcal{M}_{\mathrm{B}}$ :

$$
\mathcal{L}_{\mathbf{B}}:=\left\{a \in \mathcal{M}_{\mathbf{B}} \mid J\left(R_{a}\right) \text { is locally connected }\right\} .
$$

The basilica polynomial

$$
f_{\mathbf{B}}(z):=z^{2}-1
$$

is the only member of the quadratic family that has a superattracting 2-periodic orbit. Let $K_{\mathbf{B}}$ be the filled Julia set for $f_{\mathbf{B}}$. The following result is an analogue of the Böttcher uniformization theorem for the quadratic family. Refer to [AY] for the proof.

Proposition 2.2. Suppose $a \in \mathcal{M}_{\mathbf{B}}$. Then there exists a unique conformal map $\psi_{a}: \mathcal{A}_{a}^{\infty} \rightarrow \stackrel{\circ}{K}_{\mathbf{B}}$ such that the following diagram commutes:

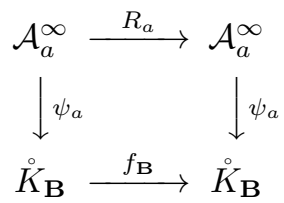

Moreover, if $B$ is a connected component of $\mathcal{A}_{a}^{\infty}$, then $\psi_{a}$ extends to a homeomorphism between $\bar{B}$ and $\psi_{a}(\bar{B})$.

Suppose for some $c \in \mathcal{L} \cap\left(\mathbb{C} \backslash L_{1 / 2}\right)$, the quadratic polynomials $f_{c}$ and $f_{\mathbf{B}}$ are conformally mateable. If $F: \hat{\mathbb{C}} \rightarrow \hat{\mathbb{C}}$ is a conformal mating of $f_{c}$ and $f_{\mathbf{B}}$, then $F$ has a superattracting 2-periodic orbit. By Proposition 2.1, $F$ can be normalized as $R_{a}$ for some $a \in \mathcal{L}_{\mathbf{B}}$.

In view of Proposition 2.2, it is natural to ask whether for every $a \in \mathcal{L}_{\mathbf{B}}$, the quadratic rational map $R_{a}$ is a conformal mating of $f_{c}$ and $f_{\mathbf{B}}$ for some $c \in \mathcal{L} \cap(\mathbb{C} \backslash$ $\left.L_{1 / 2}\right)$. It turns out this cannot be true: for some $a \in \mathcal{L}_{\mathrm{B}}$, the map $R_{a}$ is the result 


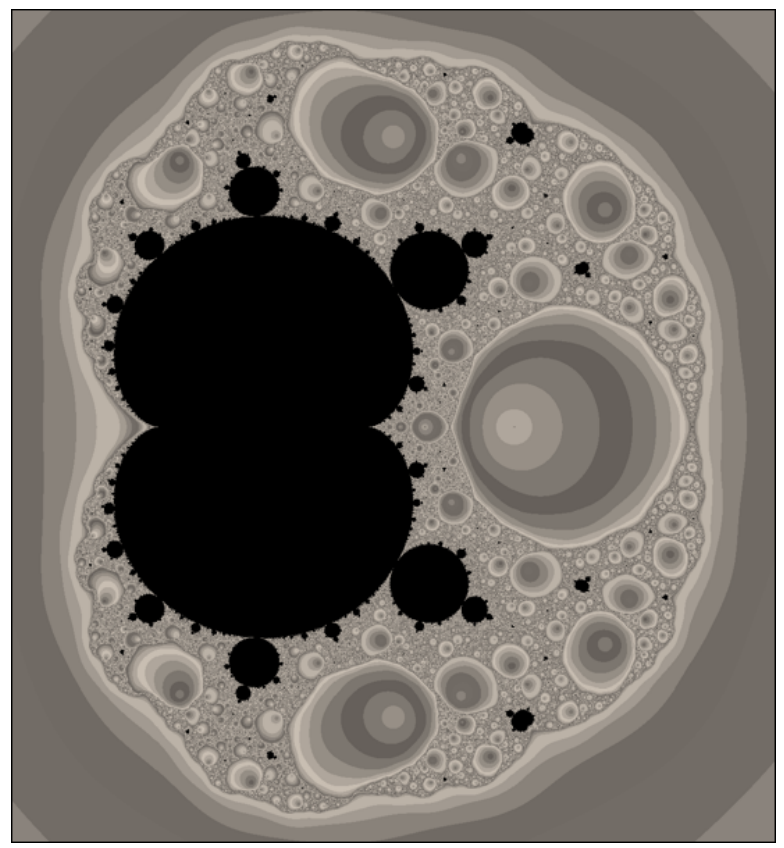

Figure 3. The non-escape locus $\mathcal{M}_{\mathbf{B}}$ for $R_{a}$ (in black). Compare with Figure 1. Note the absence of a copy of the 1/2-limb $L_{1 / 2}$ (see Example 1.5).

of a more general form of mating called mating with laminations between $f_{c}$ and $f_{\mathbf{B}}$ with $c \notin \mathcal{L}$ (see $[\mathrm{Du}]$ ). However, the following weaker statement does hold. The proof is completely analogous to the proof of Proposition 1.4, so we omit it here.

Proposition 2.3. Suppose $R_{a}$ is a conformal mating. Then $R_{a}$ is a conformal mating of $f_{c}$ and $f_{\mathbf{B}}$ for some $c \in \mathcal{L} \cap\left(\mathbb{C} \backslash L_{1 / 2}\right)$.

The principal motivation for this paper is to answer the following question:

The Main Question. Suppose $c \in \mathcal{L} \cap\left(\mathbb{C} \backslash L_{1 / 2}\right)$. Are $f_{c}$ and $f_{\mathbf{B}}$ conformally mateable? If so, is there a unique member of the basilica family that realizes their conformal mating?

We now summarize the known results on this topic.

Theorem 2.4 (Rees, Tan, Shishikura Re,Tan,, ). Suppose $c \in \mathcal{L} \cap\left(\mathbb{C} \backslash L_{1 / 2}\right)$. If $f_{c}$ is hyperbolic, then $f_{c}$ and $f_{\mathbf{B}}$ are conformally mateable. Moreover, their conformal mating is unique up to conjugacy by a Möbius map.

Theorem 2.4 is actually a corollary of a much more general result which states that two post-critically finite quadratic polynomials $f_{c_{1}}$ and $f_{c_{2}}$ are (essentially) mateable if and only if $c_{1}$ and $c_{2}$ do not belong to conjugate limbs of the Mandelbrot set. See Tan] for more details.

Theorem 2.5 (Aspenberg, Yampolsky $[\mathrm{AY}]$ ). Suppose $c \in \mathcal{L} \cap\left(\mathbb{C} \backslash L_{1 / 2}\right)$. If $f_{c}$ is at most finitely renormalizable and has no non-repelling periodic orbits, then $f_{c}$ and $f_{\mathbf{B}}$ are conformally mateable. Moreover, their conformal mating is unique up to conjugacy by a Möbius map. 
Theorem 2.6 (Dudko $[\mathrm{Du}]$ ). Suppose $c \in \mathcal{L} \cap\left(\mathbb{C} \backslash L_{1 / 2}\right)$. If $f_{c}$ is at least 4 times renormalizable, then $f_{c}$ and $f_{\mathbf{B}}$ are conformally mateable. Moreover, their conformal mating is unique up to conjugacy by a Möbius map.

Together, Theorems 2.4, 2.5 and 2.6 provide a positive answer to the main question in almost all cases. However, the parameters contained in the boundary of hyperbolic components that are not too "deep" inside the Mandelbrot set are still left unresolved. We discuss these parameters in greater detail in the next section.

\section{Matings in the Boundary of hyperbolic COMPONENTS}

Let $H$ be a hyperbolic component of $\mathcal{M} \backslash L_{1 / 2}$. By Theorem 2.4, the quadratic polynomial $f_{c}$ and the basilica polynomial $f_{\mathbf{B}}$ are conformally mateable for all $c \in H$. Our goal is to determine if this is also true for $c \in \partial H \cap \mathcal{L}$.

Choose a parameter value $c_{0} \in H$, and let $a_{0} \in \mathcal{M}_{\mathbf{B}}$ be a parameter value such that $R_{a_{0}}$ is a conformal mating of $f_{c_{0}}$ and $f_{\mathbf{B}}$. Since $R_{a_{0}}$ must be hyperbolic, $a_{0}$ is contained in some hyperbolic component $H_{\mathbf{B}}$ of $\mathcal{M}_{\mathbf{B}}$.

For all $c \in \bar{H}$, the quadratic polynomial $f_{c}$ has a non-repelling $n$-periodic orbit $\mathbf{O}_{c}:=\left\{f_{c}^{i}\left(z_{c}\right)\right\}_{i=0}^{n-1}$ for some fixed $n \in \mathbb{N}$ (see e.g. [M2]). Likewise, for all $a \in$ $\overline{H_{\mathrm{B}}}$, the quadratic rational map $R_{a}$ has a non-repelling $n$-periodic orbit $\mathcal{O}_{a}:=$ $\left\{R_{a}^{i}\left(w_{a}\right)\right\}_{i=0}^{n-1}$. Define the multiplier maps $\lambda: \bar{H} \rightarrow \overline{\mathbb{D}}$ and $\mu: \overline{H_{\mathbf{B}}} \rightarrow \overline{\mathbb{D}}$ by

$$
\lambda(c):=\left(f_{c}^{n}\right)^{\prime}\left(z_{c}\right) \quad \text { and } \quad \mu(a):=\left(R_{a}^{n}\right)^{\prime}\left(w_{a}\right) .
$$

It is known that $\lambda$ and $\mu$ are homeomorphisms which are conformal on the interior of their domains (see M2]).

The following result can be proved using a standard application of quasiconformal surgery (see Chapter 4 in $[\overline{B F}]$ ).

Proposition 3.1. Define a homeomorphism $\phi_{H}: \bar{H} \rightarrow \overline{H_{\mathbf{B}}}$ by

$$
\phi_{H}:=\mu^{-1} \circ \lambda \text {. }
$$

Then for all $c \in H$, the quadratic rational map $R_{\phi_{H}(c)}$ is a conformal mating of $f_{c}$ and $f_{\mathbf{B}}$.

Our goal is to extend the statement of Proposition 3.1 to the boundary of $H$ where possible.

Consider $c \in \partial H$, and let $a=\phi_{H}(c) \in \partial H_{\mathbf{B}}$. The multiplier of $\mathbf{O}_{c}$ and $\mathcal{O}_{a}$ is equal to $e^{2 \pi \theta i}$ for some $\theta \in \mathbb{R} / \mathbb{Z}$. The number $\theta$ is referred to as the rotation number. If $\theta$ is rational, then $\mathbf{O}_{c}$ and $\mathcal{O}_{a}$ are parabolic. In this case, an application of trans-quasiconformal surgery due to Haïssinsky implies the following result (see $[\mathrm{Ha}]$ ).

Theorem 3.2. Suppose that the rotation number $\theta$ is rational, so that $\mathbf{O}_{c}$ and $\mathcal{O}_{a}$ are parabolic. Then $f_{c}$ and $f_{\mathbf{B}}$ are conformally mateable, and $R_{a}$ is the unique member of the basilica family that realizes their conformal mating.

If $\theta$ is irrational, then $\mathbf{O}_{c}$ is either Siegel or Cremer. In the latter case, it is known that the Julia set $J_{c}$ for $f_{c}$ is non-locally connected (see e.g. M1]). This means that the formal mating of $f_{c}$ and $f_{\mathbf{B}}$ cannot be defined, and hence, they are not conformally mateable. 
For our discussion of the Siegel case, we first recall a classical result of Siegel $[\mathbf{S}]$. An irrational number $x$ is said to be Diophantine of order $\kappa$ if there exists a fixed constant $\epsilon>0$ such that for all $\frac{p}{q} \subset \mathbb{Q}$, the following inequality holds:

$$
\left|x-\frac{p}{q}\right| \geq \frac{\epsilon}{q^{\kappa}} .
$$

The set of all irrational numbers that are Diophantine of order $\kappa$ is denoted $\mathcal{D}(\kappa)$. The smallest possible value of $\kappa$ such that $\mathcal{D}(\kappa)$ is non-empty is 2 (see [M1]).

Theorem 3.3 (Siegel [S]). Let $f: U \rightarrow V$ be an analytic function. Suppose $f$ has an indifferent periodic orbit $\mathcal{O}$ with an irrational rotation number $\theta$. If $\theta \in \mathcal{D}(\kappa)$ for some $\kappa \geq 2$, then $\mathcal{O}$ is a Siegel orbit.

There is a classical connection between Diophantine classes and continued fraction approximations (see e.g. [M1]). In particular, if

$$
x=\frac{1}{a_{1}+\frac{1}{a_{2}+\ldots}}
$$

is the continued fraction representation of $x$, then $x \in \mathcal{D}(2)$ if and only if all the $a_{i}$ 's are uniformly bounded. In view of this, we say that the numbers contained in $\mathcal{D}(2)$ are of bounded type. Siegel quadratic polynomials of bounded type are prominently featured in the study of renormalization (see e.g. [P, $\mathrm{Mc}, \mathrm{Y} 1, \mathrm{Y} 2]$ ).

Theorem 3.4 (Peterson $[\mathrm{P}]$ ). Suppose a quadratic polynomial $f_{c}$ has an indifferent periodic orbit with an irrational rotation number of bounded type. Then $f_{c}$ has a locally connected Julia set $J_{c}$.

In this paper, we present a positive answer to the main question (stated in Section 2 for quadratic polynomials $f_{\mathbf{S}}$ that have an indifferent fixed point with an irrational rotation number of bounded type. Note that by Theorem 3.3 , the indifferent fixed point is Siegel, and by Theorem 3.4, the formal mating of $f_{\mathbf{S}}$ and $f_{\mathbf{B}}$ is well defined.

The solution to the uniqueness part of the main question is elementary.

Proposition 3.5. Suppose $\lambda \in \overline{\mathbb{D}}$. Then there exists a unique $c \in \mathcal{M}$ (resp. $a \in \mathcal{M}_{\mathbf{B}}$ ) such that $f_{c}$ (resp. $R_{a}$ ) has a bounded non-repelling fixed point $z_{0} \neq \infty$ with multiplier $\lambda$.

Proof. Suppose $f_{c}$ has a fixed point $z_{0} \neq \infty$ with multiplier $\lambda \in \mathbb{C}$. It is easy to check that the value of $c$ is given by

$$
c=\frac{\lambda}{2}-\frac{\lambda^{2}}{4}
$$

Hence, $c$ is uniquely determined.

Likewise, suppose $R_{a}$ has a fixed point with multiplier $\lambda \in \mathbb{C}$. Then the value of $a$ is given by

$$
a=-\frac{8 \lambda}{(\lambda-1)^{3}} .
$$

Hence, $a$ is uniquely determined.

Our main results are stated below. 
Theorem A. Suppose $\nu \in \mathbb{R} \backslash \mathbb{Q}$ is of bounded type. Let $R_{a_{\nu}}$ with $a_{\nu} \in \mathcal{M}_{\mathbf{B}}$ be the unique member of the basilica family that has a Siegel fixed point $z_{0}$ with rotation number $\nu$. Let $S$ be the fixed Siegel disc containing $z_{0}$. Then $S$ is a quasidisk, and contains the critical point -1 in its boundary.

Theorem B. Suppose $\nu \in \mathbb{R} \backslash \mathbb{Q}$ is of bounded type. Let $f_{\mathbf{S}}$ be the unique member of the quadratic family that has a Siegel fixed point with rotation number $\nu$. Then $f_{\mathbf{S}}$ and $f_{\mathbf{B}}$ are conformally mateable, and $R_{a_{\nu}}$ is the unique member of the basilica family that realizes their conformal mating.

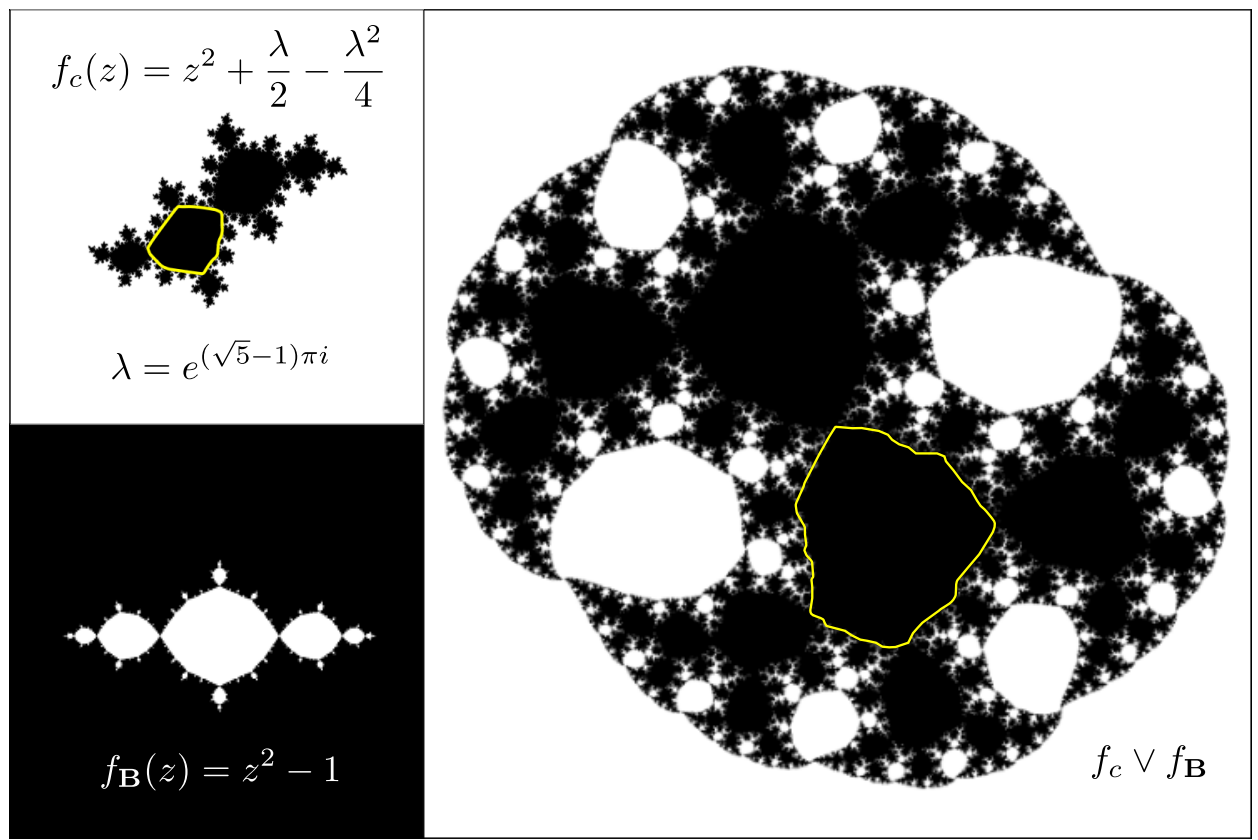

Figure 4. The Siegel polynomial $f_{c}$ with $c=\frac{\lambda}{2}-\frac{\lambda^{2}}{4}$ and $\lambda=$ $e^{(\sqrt{5}-1) \pi i}$ mated with the basilica polynomial $f_{\mathbf{B}}$. The Siegel disc is highlighted.

\section{The construction of a Blaschke product model AND THE PROOF OF THEOREM A}

Consider the Blaschke product

$$
F_{a, b}(z):=-\frac{1}{e^{i \theta}} \frac{z(z-a)(z-b)}{(1-\bar{a} z)(1-\bar{b} z)},
$$

where $a b=r e^{i \theta}$ with $r \in \mathbb{R}^{+}$and $\theta \in[0,2 \pi)$. Note that 0 is a fixed point with multiplier $-r$.

Lemma 4.1. For any value of $r$ and $\theta$, the parameters $a=a(r, \theta)$ and $b=b(r, \theta)$ can be chosen such that $F_{a, b}$ has a double critical point at 1. 
Proof. Let

Then

$$
F_{a, b}^{\prime}(z)=\frac{P(z)}{Q(z)}
$$

$$
F_{a, b}^{\prime \prime}(z)=\frac{P^{\prime}(z) Q(z)-P(z) Q^{\prime}(z)}{Q(z)^{2}} .
$$

Thus, the condition

$$
F_{a, b}^{\prime}(1)=F_{a, b}^{\prime \prime}(1)=0
$$

is equivalent to

$$
P(1)=P^{\prime}(1)=0 .
$$

A straightforward computation shows that

$$
P(z)=\bar{\kappa} z^{4}-2 \bar{\zeta} z^{3}+\left(3-|\kappa|^{2}+|\zeta|^{2}\right) z^{2}-2 \zeta z+\kappa,
$$

where

$$
\kappa:=a b \quad \text { and } \quad \zeta:=a+b .
$$

Thus, $F_{a, b}$ has a double critical point at 1 if the following two equations are satisfied:

$$
\begin{aligned}
& 2 \kappa-3 \zeta+\left(3-|\kappa|^{2}+|\zeta|^{2}\right)=\bar{\zeta}, \\
& 3 \kappa-2 \zeta+\left(3-|\kappa|^{2}+|\zeta|^{2}\right)=\bar{\kappa} .
\end{aligned}
$$

Subtracting (4.1) from (4.2), we see that

$$
\kappa-\zeta=\bar{\kappa}-\bar{\zeta} \text {. }
$$

Substituting $\kappa=x+i y$ and $\zeta=u+i y$ into (4.1), we obtain

$$
u^{2}-4 u+\left(2 x-x^{2}+3\right)=0 .
$$

The equation (4.3) has two solutions: $u=-x+3$ and $u=x+1$. The first solution corresponds to the relation

$$
\zeta=-\bar{\kappa}+3 .
$$

Therefore, by choosing $a$ and $b$ to be the solutions of

$$
z^{2}+\left(r e^{-i \theta}-3\right) z+r e^{i \theta}=0
$$

we ensure that the map $F_{a, b}$ has a double critical point at 1 .

Lemma 4.2. Let $a=a(r, \theta)$ and $b=b(r, \theta)$ satisfy the condition in Lemma 4.1. Then for all $r>1$ sufficiently close to 1 , there exists a local holomorphic change of coordinates $\phi$ at 0 so that the map $G:=\phi^{-1} \circ F_{a, b}^{2} \circ \phi$ takes the form

$$
G(z)=r^{2} z\left(1+z^{2}+\mathcal{O}(z)\right) .
$$

Proof. Expanding $F_{a, b}(z)$ as a power series around 0, we have

$$
F_{a, b}(z)=-r z+\lambda z^{2}+\mathcal{O}\left(z^{3}\right)
$$

for some $\lambda=\lambda(r, \theta)$ depending continuously on $r$ and $\theta$. Define

$$
\psi_{\mu}(z):=z+\mu z^{2} \quad \text { for } \quad \mu \in \mathbb{C} .
$$

A straightforward computation shows that

$$
H(z):=\psi_{\mu}^{-1} \circ F_{a, b} \circ \psi_{\mu}(z)=-r z+(\lambda+(1+r) \mu) z^{2}+\mathcal{O}\left(z^{3}\right) .
$$

Thus, by choosing $\mu=\frac{-\lambda}{1+r}$, we have

$$
H(z)=-r z\left(1+\nu z^{2}+\mathcal{O}\left(z^{3}\right)\right)
$$


for some $\nu=\nu(r, \theta)$ depending continuously on $r$ and $\theta$.

Observe that the second iterate of $H$ is equal to

$$
H^{2}(z)=r^{2} z\left(1+\left(1+r^{2}\right) \nu z^{2}+\mathcal{O}\left(z^{3}\right)\right) .
$$

When $r=1$, the point 0 is a parabolic fixed point of multiplicity 2 . This means that $\nu(1, \theta)$ cannot be equal to zero for all $\theta \in[0,2 \pi)$. Hence, for some $\epsilon>0$ sufficiently small, $\nu(r, \theta)$ is not equal to zero for all $r \in(1,1+\epsilon)$ and $\theta \in[0,2 \pi)$. After one more change of coordinates, we arrive at

$$
G(z):=\sqrt{\left(1+r^{2}\right) \nu} \cdot H^{2}\left(\frac{z}{\sqrt{\left(1+r^{2}\right) \nu}}\right)=r^{2} z\left(1+z^{2}+\mathcal{O}\left(z^{3}\right)\right) .
$$

Lemma 4.3. Let $a=a(r, \theta)$ and $b=b(r, \theta)$ satisfy the condition in Lemma 4.1. Then for all $r>1$ sufficiently close to 1 , the Blaschke product $F_{a, b}$ has an attracting 2-periodic orbit near 0.

Proof. Consider the map $G:=\phi^{-1} \circ F_{a, b}^{2} \circ \phi$ defined in Lemma 4.2. We prove that $G$ has two attracting fixed points near 0 .

Observe that $G$ satisfies

$$
|G(z)|=r^{2}|z|\left(1+\operatorname{Re}\left(z^{2}\right)+(\text { higher terms })\right)
$$

and

$$
\arg (G(z))=\arg (z)+\operatorname{Im}\left(z^{2}\right)+\text { (higher terms) } .
$$

Consider the wedge shaped regions

$$
V_{\epsilon}^{+}:=\left\{\rho e^{2 \pi i t} \in \mathbb{C} \mid 0 \leq \rho \leq \epsilon, \frac{3}{16} \leq t \leq \frac{5}{16}\right\}
$$

and

$$
V_{\epsilon}^{-}:=-V_{\epsilon}^{+} \text {. }
$$

It is easily checked that $G\left(V_{\epsilon}^{+}\right) \subset V_{\epsilon}^{+}$and $G\left(V_{\epsilon}^{-}\right) \subset V_{\epsilon}^{-}$. Since 0 is the only fixed point on the boundary of these regions, and it is repelling, $V_{\epsilon}^{+}$and $V_{\epsilon}^{-}$must each contain an attracting fixed point for $G$.

Theorem 4.4. Given any angle $\nu \in[0,2 \pi)$, there exists a Blaschke product $F_{\nu}$ that satisfies the following three properties:

(i) There exists a superattracting 2-periodic orbit $\mathcal{O}=\left\{\infty, F_{\nu}(\infty)\right\}$ with a critical point at $\infty$.

(ii) The rotation number of the map $\left.F_{\nu}\right|_{\partial \mathbb{D}}$ is equal to $\nu$.

(iii) The point 1 is a double critical point.

Proof. The family of Blaschke products $\left\{F_{a, b}\right\}$ that satisfy Lemmas 4.1 and 4.3 are continuously parameterized by $r$ and $\theta$. Let $\rho(r, \theta)$ denote the rotation number of the map $\left.F_{a, b}\right|_{\partial \mathbb{D}}$. In $\mathrm{YZ}$, it is proved that $\rho(1, \cdot)$ is not nullhomotopic. By continuity, $\rho(r, \cdot)$ is also not nullhomotopic. Thus, for any angle $\nu \in[0,2 \pi)$, there exists $\theta$ such that $\rho(r, \theta)=\nu$.

So far, we have proved the existence of a Blaschke product $F_{a, b}$ that has an attracting 2-periodic orbit near zero, has a double critical point at 1 , and whose restriction to $\partial \mathbb{D}$ has rotation number equal to $\nu$. A standard application of quasiconformal surgery turns the attracting 2-periodic orbits of $F_{a, b}$ into superattracting orbits (the surgery must be symmetric with respect to the unit circle to ensure that the resulting map is also a Blaschke product). Then after conjugating by the appropriate Blaschke factor, we obtain the desired map $F_{\nu}$. 


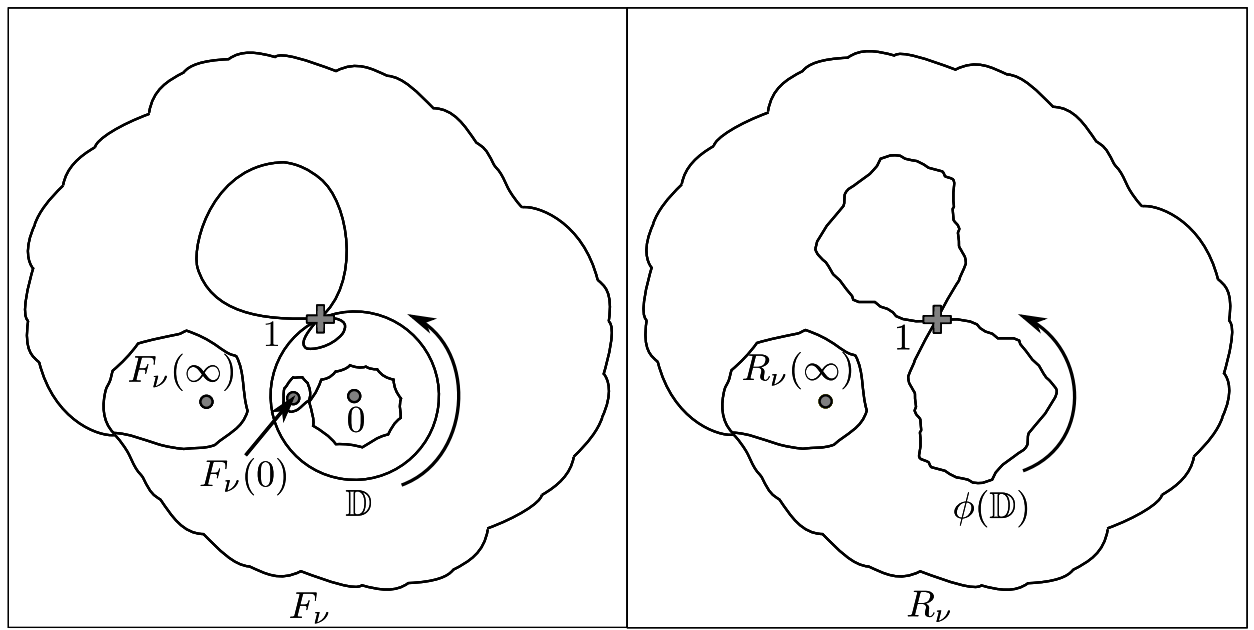

FiguRE 5. An illustration of the quasiconformal surgery in Theorem 4.5. The image of $\mathbb{D}$ under the quasiconformal map $\phi$ is a Siegel disc for $R_{\nu}$. Also note that the double critical point for $F_{\nu}$ (represented by a cross) becomes a single critical point for $R_{\nu}$.

Theorem 4.5. Suppose $\nu$ is irrational and of bounded type. Let $F_{\nu}$ be the Blaschke product constructed in Theorem 4.4. Then there exists a quadratic rational function $R_{\nu}$ and quasiconformal maps $\psi: \mathbb{D} \rightarrow \mathbb{D}$, and $\phi: \hat{\mathbb{C}} \rightarrow \hat{\mathbb{C}}$ such that $\psi(1)=1$, $\phi(1)=1, \phi(\infty)=\infty$, and $\phi(\psi(0))=0$, and

$$
R_{\nu}(z)= \begin{cases}\phi \circ \psi \circ \operatorname{Rot}_{\nu} \circ \psi^{-1} \circ \phi^{-1}(z) & : \text { if } z \in \phi(\mathbb{D}) \\ \phi \circ F_{\nu} \circ \phi^{-1}(z) & : \text { if } z \in \hat{\mathbb{C}} \backslash \phi(\mathbb{D}) .\end{cases}
$$

Proof. Since $\nu$ is of bounded type, there exists a unique homeomorphism $\psi: \partial \mathbb{D} \rightarrow$ $\partial \mathbb{D}$ such that $\psi(1)=1$, and

$$
\psi \circ \operatorname{Rot}_{\nu} \circ \psi^{-1}=\left.F_{\nu}\right|_{\partial \mathbb{D}}
$$

Moreover, $\psi$ extends to a quasiconformal map on $\mathbb{D}$.

Define

$$
g(z)= \begin{cases}\psi \circ \operatorname{Rot}_{\nu} \circ \psi^{-1}(z) & : \text { if } z \in \mathbb{D}, \\ F_{\nu}(z) & : \text { if } z \in \hat{\mathbb{C}} \backslash \mathbb{D} .\end{cases}
$$

By construction, $g$ is continuous.

To obtain a holomorphic map with the same dynamics as $g$, we define and integrate a new complex structure $\mu$ on $\hat{\mathbb{C}}$. Start by defining $\mu$ on $\mathbb{D}$ as the pullback of the standard complex structure $\sigma_{0}$ by $\psi^{-1}$. Next, pull back $\mu$ on $\mathbb{D}$ by the iterates of $g$ to define $\mu$ on the iterated preimages of $\mathbb{D}$. Finally, extend $\mu$ to the rest of $\hat{\mathbb{C}}$ as the standard complex structure $\sigma_{0}$.

Let $\phi: \hat{\mathbb{C}} \rightarrow \hat{\mathbb{C}}$ be the unique solution of the Beltrami equation

$$
\partial_{\bar{z}} \phi(z)=\mu(z) \partial_{z} \phi(z)
$$

such that $\phi(1)=1, \phi(\infty)=\infty$ and $\phi(\psi(0))=0$. Then the map

$$
R_{\nu}:=\phi \circ g \circ \phi^{-1}
$$

gives us the desired quadratic rational function. 
Proof of Theorem A. Consider the quadratic rational function $R_{\nu}$ constructed in Theorem 4.5. Observe that $R_{\nu}$ satisfies the following three properties:

(i) There exists a superattracting 2-periodic orbit $\left\{\infty, R_{\nu}(\infty)\right\}$ with a critical point at $\infty$.

(ii) The image of $\mathbb{D}$ under the quasiconformal map $\phi$ is a Siegel disc with rotation number $\nu$.

(iii) The point 1 is a critical point, and is contained in $\partial \phi(\mathbb{D})$.

Clearly, the critical value $R_{\nu}(\infty)$ is not equal to the critical point 1 . The theorem now follows from Proposition 2.1.

\section{The COnstruction of BUbBle RAYs}

5.1. For the basilica polynomial. Consider the basilica polynomial

$$
f_{\mathbf{B}}(z):=z^{2}-1 \text {. }
$$

Note that $f_{\mathbf{B}}$ has a superattracting 2-periodic orbit $\{0,-1\}$, and hence, is hyperbolic. Denote the Julia set and the filled Julia set for $f_{\mathbf{B}}$ by $J_{\mathbf{B}}$ and $K_{\mathbf{B}}$ respectively. The following is a consequence of the hyperbolicity of $f_{\mathbf{B}}$ (see e.g. [M1]).

Proposition 5.1. The Julia set $J_{\mathbf{B}}$ for $f_{\mathbf{B}}$ is locally connected.

A connected component of $\mathbf{B}:=\dot{K}_{\mathbf{B}}$ is called a $\boldsymbol{b u b b l e}$. Let $\mathbf{B}_{0}$ be the bubble containing the critical point 0 . We have

$$
\mathbf{B}=\bigcup_{n=0}^{\infty} f_{\mathbf{B}}^{-n}\left(\mathbf{B}_{0}\right) \text {. }
$$

Let $B \subset \mathbf{B}$ be a bubble. The generation of $B$, denoted by $\operatorname{gen}(B)$, is defined to be the smallest number $n \in \mathbb{N}$ such that $f_{\mathbf{B}}^{n}(B)=\mathbf{B}_{0}$. The center of $B$ is the unique point $z \in B$ that is mapped to 0 under $f_{\mathbf{B}}^{\operatorname{gen}(B)}$.

Proposition 5.2. There exists a unique repelling fixed point $\mathbf{b}$ contained in $\partial \mathbf{B}_{0}$.

Note that the repelling fixed point $\mathbf{b}$ in Proposition 5.2 is the $\alpha$-fixed point of $f_{\mathbf{B}}$ (see [M2]).

Let $b \in J_{\mathbf{B}}$ be an iterated preimage of $\mathbf{b}$. The generation of $b$, denoted by $\operatorname{gen}(b)$, is defined to be the smallest number $n \in \mathbb{N}$ such that $f_{\mathbf{B}}^{n}(b)=\mathbf{b}$. Suppose $b$ is contained in the boundary of some bubble $B$. If the generation of $b$ is the smallest among all iterated preimages of $\mathbf{b}$ that are contained in $\partial B$, then $b$ is called the root of $B$. It is easy to see that every bubble has a unique root.

Proposition 5.3. Let $b \in J_{\mathbf{B}}$ be an iterated preimage of $\mathbf{b}$. Then there are exactly two bubbles $B_{1}$ and $B_{2}$ in $\mathbf{B}$ which contain $b$ in their closures. Moreover, we have

$$
\overline{B_{1}} \cap \overline{B_{2}}=\{b\} .
$$

Proof. There are exactly two bubbles, $\mathbf{B}_{0}$ and $f_{\mathbf{B}}\left(\mathbf{B}_{0}\right)$, that contain $\mathbf{b}$ in their closures. Moreover, we have

$$
\overline{\mathbf{B}_{0}} \cap \overline{f_{\mathbf{B}}\left(\mathbf{B}_{0}\right)}=\{\mathbf{b}\} .
$$

There exists a neighborhood $N$ containing $b$ such that $N$ is mapped conformally onto a neighborhood of $\mathbf{b}$ by $f_{\mathbf{B}}^{\text {gen }(b)}$. The result follows. 
Let $b \in J_{\mathbf{B}}$ be an iterated preimage of $\mathbf{b}$, and let $B_{1}$ and $B_{2}$ be the two bubbles that contain $b$ in their closures. Suppose gen $\left(B_{1}\right)<\operatorname{gen}\left(B_{2}\right)$. Then $B_{1}$ and $B_{2}$ are referred to as the parent and the child at $b$ respectively. Note that $b$ must be the root of $B_{2}$.

Consider a set of bubbles $\left\{B_{i}\right\}_{i=0}^{n}$ in $\mathbf{B}$, and a set of iterated preimages $\left\{b_{i}\right\}_{i=0}^{n}$ of $\mathbf{b}$ such that the following properties are satisfied:

(i) $B_{0}=\mathbf{B}_{0}$ and $b_{0}=\mathbf{b}$, and

(ii) for $1 \leq i \leq n$, the bubbles $B_{i-1}$ and $B_{i}$ are the parent and the child at $b_{i}$ respectively.

The set

$$
\mathcal{R}^{\mathbf{B}}:=\overline{f_{\mathbf{B}}\left(\mathbf{B}_{0}\right)} \cup\left(\bigcup_{i=0}^{n} \overline{B_{i}}\right)
$$

is called a bubble ray for $f_{\mathbf{B}}$ (the inclusion of $\overline{f_{\mathbf{B}}\left(\mathbf{B}_{0}\right)}$ is to ensure that a bubble ray is mapped to a bubble ray). For conciseness, we use the notation $\mathcal{R}^{\mathbf{B}} \sim\left\{B_{i}\right\}_{i=0}^{n}$. The bubble ray $\mathcal{R}^{\mathrm{B}}$ is said to be finite or infinite according to whether $n<\infty$ or $n=\infty$. Lastly, $\left\{b_{i}\right\}_{i=0}^{n}$ is called the set of attachment points for $\mathcal{R}^{\mathbf{B}}$.

Proposition 5.4. If $B \subset \mathbf{B}$ is a bubble, then there exists a unique finite bubble ray $\mathcal{R}^{\mathbf{B}} \sim\left\{B_{i}\right\}_{i=0}^{n}$ such that $B_{n}=B$. Consequently, if $\mathcal{R}_{1}^{\mathbf{B}} \sim\left\{B_{i}^{1}\right\}_{i=0}^{n}$ and $\mathcal{R}_{2}^{\mathbf{B}} \sim$ $\left\{B_{i}^{2}\right\}_{i=0}^{m}$ are two bubble rays, then there exists $N \geq 0$ such that $B_{i}^{1}=B_{i}^{2}$ for all $i \leq N$, and $B_{i}^{1} \neq B_{i}^{2}$ for all $i>N$.

Proof. We can construct a finite bubble ray ending in $B$ as follows. First, let $\tilde{B}_{0}=B$. Next, let $\tilde{b}_{0}$ be the root of $\tilde{B}_{0}$, and let $\tilde{B}_{1}$ be the parent of $\tilde{B}_{0}$ at $\tilde{b}_{0}$. Proceeding inductively, we obtain a sequence of bubbles $\tilde{B}_{0}, \tilde{B}_{1}, \tilde{B}_{2}, \ldots$, and a sequence of roots $\tilde{b}_{0}, \tilde{b}_{1}, \tilde{b}_{2}, \ldots$, such that $\tilde{B}_{i+1}$ is the parent of $\tilde{B}_{i}$ at $\tilde{b}_{i}$. Since $\operatorname{gen}\left(\tilde{B}_{i+1}\right)$ is strictly less than gen $\left(\tilde{B}_{i}\right)$, this sequence must terminate at $\tilde{B}_{n}=\mathbf{B}_{0}$ for some $n \geq 0$. Then $\mathcal{R}^{\mathbf{B}} \sim\left\{\tilde{B}_{n-i}\right\}_{i=0}^{n}$ is the desired finite bubble ray. The uniqueness of $\mathcal{R}^{\mathrm{B}}$ follows from the uniqueness of the root of a bubble and Proposition 5.3 .

Let $\mathcal{R}^{\mathbf{B}} \sim\left\{B_{i}\right\}_{i=0}^{\infty}$ be an infinite bubble ray. We say that $\mathcal{R}^{\mathbf{B}}$ lands at $z \in J_{\mathbf{B}}$ if the sequence of bubbles $\left\{B_{i}\right\}_{i=0}^{\infty}$ converges to $z$ in the Hausdorff topology. The following result is a consequence of the hyperbolicity of $f_{\mathbf{B}}$ (see $[\mathrm{DH}]$ ).

Proposition 5.5. There exists $0<s<1$, and $C>0$ such that for every bubble $B \subset \mathbf{B}$, we have

$$
\operatorname{diam}(B)<C s^{\operatorname{gen}(B)} .
$$

Consequently, every infinite bubble ray for $f_{\mathbf{B}}$ lands.

Denote the attracting basin of infinity for $f_{\mathbf{B}}$ by $\mathbf{A}_{\mathbf{B}}^{\infty}$. Let

$$
\phi_{\mathbf{A}_{\mathbf{B}}^{\infty}}: \mathbf{A}_{\mathbf{B}}^{\infty} \rightarrow \mathbb{C} \backslash \overline{\mathbb{D}}
$$

and

$$
\phi_{\mathbf{B}_{0}}: \mathbf{B}_{0} \rightarrow \mathbb{D}
$$

be the Böttcher uniformization of $f_{\mathbf{B}}$ on $\mathbf{A}_{\mathbf{B}}^{\infty}$ and $\mathbf{B}_{0}$ respectively. Using $\phi_{\mathbf{A}_{\mathbf{B}}^{\infty}}$ and $\phi_{\mathbf{B}_{0}}$, we can encode the dynamics of bubble rays for $f_{\mathbf{B}}$ in two different ways: via external angles, and via bubble addresses.

Suppose that $\mathcal{R}^{\mathbf{B}}$ is an infinite bubble ray, and let $z \in J_{\mathbf{B}}$ be its landing point. Then there exists a unique external ray

$$
\mathcal{R}_{-t}^{\infty}:=\left\{\arg \left(\phi_{\mathbf{A}_{\mathbf{B}}^{\infty}}\right)=-t\right\}
$$


which lands at $z$ (note that arg stands for the argument of a complex number- e.g. if $w=r e^{2 \pi i \theta}$, then $\left.\arg (w)=\theta\right)$. The external angle of $\mathcal{R}^{\mathbf{B}}$ is defined to be $t$. Henceforth, the infinite bubble ray with external angle $t$ will be denoted $\mathcal{R}_{t}^{\mathbf{B}}$.

Let $b \in \partial \mathbf{B}_{0}$ be an iterated preimage of $\mathbf{b}$. Define

$$
\operatorname{adr}(b):=\arg \left(\phi_{\mathbf{B}_{0}}(b)\right) \text {. }
$$

If $b^{\prime}$ is an interated preimage of $\mathbf{b}$ and $b^{\prime} \notin \partial \mathbf{B}_{0}$, then there exists a unique bubble $B \subset \mathbf{B}$ such that $B$ is the parent at $b^{\prime}$. In this case, define

$$
\operatorname{adr}\left(b^{\prime}\right):=\operatorname{adr}\left(f_{\mathbf{B}}^{\operatorname{gen}(B)}\left(b^{\prime}\right)\right) .
$$

Let $\mathcal{R}^{\mathbf{B}}$ be a bubble ray and let $\left\{b_{i}\right\}_{i=0}^{n}$ be the set of attachment points for $\mathcal{R}^{\mathbf{B}}$. The bubble address of $\mathcal{R}^{\mathrm{B}}$ is defined to be

$$
\operatorname{adr}\left(\mathcal{R}^{\mathbf{B}}\right):=\left(\operatorname{adr}\left(b_{0}\right), \operatorname{adr}\left(b_{1}\right), \ldots, \operatorname{adr}\left(b_{n}\right)\right),
$$

where the tuple is interpreted to be infinite if $\mathcal{R}^{\mathbf{B}}$ is an infinite bubble ray.

If $B \subset \mathbf{B}$ is a bubble, then by Proposition 5.4, there exists a unique finite bubble ray $\mathcal{R}^{\mathbf{B}} \sim\left\{B_{i}\right\}_{i=0}^{n}$ such that $B=B_{n}$. The bubble address of $B$ is defined to be

$$
\operatorname{adr}(B):=\operatorname{adr}\left(\mathcal{R}^{\mathbf{B}}\right) .
$$

5.2. For the Siegel polynomial. Suppose $\nu \in \mathbb{R} \backslash \mathbb{Q}$ is of bounded type, and let $f_{\mathbf{S}}$ be the unique member of the quadratic family that has a Siegel fixed point $z_{0}$ with rotation number $\nu$. Denote the Siegel disc, the Julia set and the filled Julia set for $f_{\mathbf{S}}$ by $\mathbf{S}_{0}, J_{\mathbf{S}}$ and $K_{\mathbf{S}}$ respectively. By Theorem 3.4, $J_{\mathbf{S}}$ is locally connected. A quasiconformal surgery procedure due to Douady, Ghys, Herman, and Shishikura (see e.g. $[\mathrm{P}$ ) implies the following:

Theorem 5.6. The Siegel disc $\mathbf{S}_{0}$ is a quasidisc whose boundary contains the critical point 0 .

A connected component of $\mathbf{S}:=\check{K}_{\mathbf{S}}^{\circ}$ is called a $\boldsymbol{b u b b l e}$. Note that

$$
\mathbf{S}=\bigcup_{n=0}^{\infty} f_{\mathbf{S}}^{-n}\left(\mathbf{S}_{0}\right) \text {. }
$$

Let $S \subset \mathbf{S}$ be a bubble. The generation of $S$, denoted by $\operatorname{gen}(S)$, is defined to be the smallest number $n \in \mathbb{N}$ such that $f_{\mathbf{S}}^{n}(S)=\mathbf{S}_{0}$. The center of $S$ is the unique point $z \in S$ that is mapped to the Siegel fixed point $z_{0}$ by $f_{\mathbf{S}}^{\operatorname{gen}(S)}$.

Let $s \in J_{\mathbf{S}}$ be an iterated preimage of the critical point 0 . The generation of $s$, denoted by gen $(s)$, is defined to be the smallest number $n \in \mathbb{N}$ such that $f_{\mathbf{S}}^{n}(s)=0$.

Proposition 5.7. Let $s \in J_{\mathbf{S}}$ be an iterated preimage of the critical point 0 . Then there are exactly two bubbles $S_{1}$ and $S_{2}$ in $\mathbf{S}$ which contain $s$ in their closure. Moreover, we have

$$
\overline{S_{1}} \cap \overline{S_{2}}=\{s\} .
$$

The construction of a bubble ray $\mathcal{R}^{\mathbf{S}}$ for $f_{\mathbf{S}}$ is completely analogous to the construction of a bubble ray $\mathcal{R}^{\mathbf{B}}$ for $f_{\mathbf{B}}$.

Proposition 5.8. If $S \subset \mathbf{S}$ is a bubble, then there exists a unique finite bubble ray $\mathcal{R}^{\mathbf{S}} \sim\left\{S_{i}\right\}_{i=0}^{n}$ such that $S_{n}=S$. Consequently, if $\mathcal{R}_{1}^{\mathbf{S}} \sim\left\{S_{i}^{1}\right\}_{i=0}^{n}$ and $\mathcal{R}_{2}^{\mathbf{S}} \sim\left\{S_{i}^{2}\right\}_{i=0}^{m}$ are two bubble rays, then there exists $M \geq 0$ such that $S_{i}^{1}=S_{i}^{2}$ for all $i \leq M$, and $S_{i}^{1} \neq S_{i}^{2}$ for all $i>M$. 


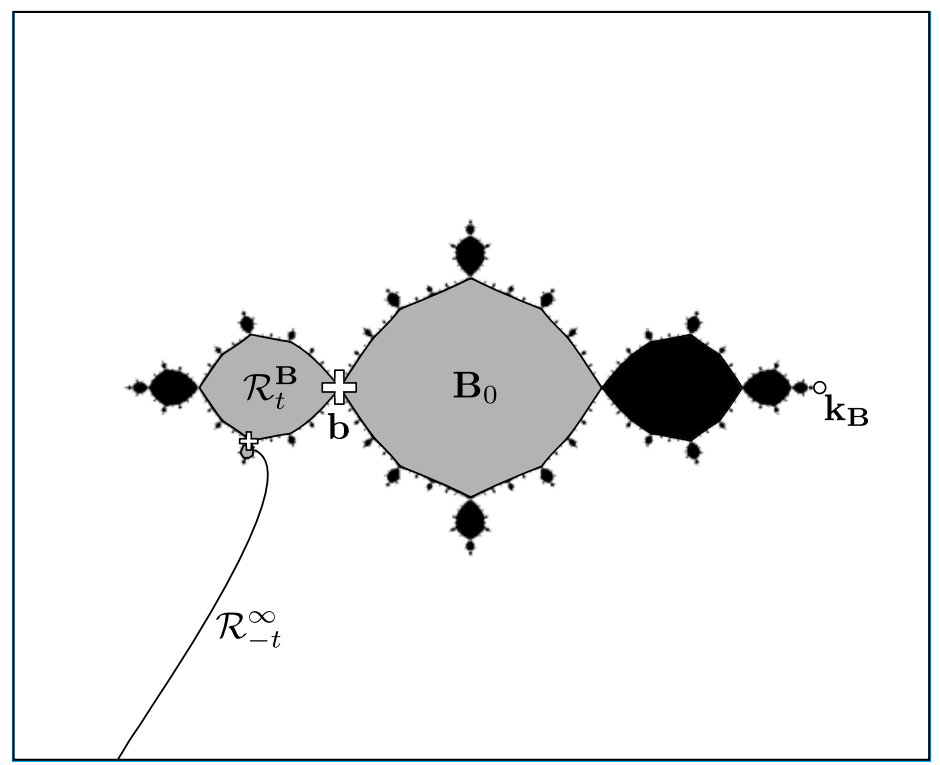

FiguRE 6 . The infinite bubble ray $\mathcal{R}_{t}^{\mathbf{B}}$ with $t \approx 0.354841$ for the basilica polynomial $f_{\mathbf{B}}$. The bubbles contained in $\mathcal{R}_{t}^{\mathbf{B}}$ are colored in light gray. The white crosses represent the set of attachment points for $\mathcal{R}_{t}^{\mathrm{B}}$.

The following proposition is a consequence of complex a priori bounds due to Yampolsky (see [Y1]). It is proved in the same way as Proposition 8.5.

Proposition 5.9. Every infinite bubble ray $\mathcal{R}^{\mathrm{S}}$ for $f_{\mathbf{S}}$ lands.

Denote the attracting basin of infinity for $f_{\mathbf{S}}$ by $\mathbf{A}_{\mathbf{S}}^{\infty}$. Let

$$
\phi_{\mathbf{A}_{\mathbf{S}}^{\infty}}: \mathbf{A}_{\mathbf{S}}^{\infty} \rightarrow \mathbb{C} \backslash \overline{\mathbb{D}}
$$

be the Böttcher uniformization of $f_{\mathbf{S}}$ on $\mathbf{A}_{\mathbf{S}}^{\infty}$.

Suppose $\mathcal{R}^{\mathbf{S}}$ is an infinite bubble ray, and let $z \in J_{\mathbf{S}}$ be its landing point. Then there exists a unique external ray

$$
\mathcal{R}_{t}^{\infty}:=\left\{\arg \left(\phi_{\mathbf{A}_{\mathbf{S}}^{\infty}}\right)=t\right\}
$$

which lands at $z$. The external angle of $\mathcal{R}^{\mathbf{S}}$ is defined to be $t$. Henceforth, the infinite bubble ray with external angle $t$ will be denoted $\mathcal{R}_{t}^{\mathbf{S}}$.

Let $s \in \partial \mathbf{S}_{0}$ be an iterated preimage of 0 . Define

$$
\operatorname{adr}(s):=\operatorname{gen}(s) .
$$

The bubble address of a bubble $S \subset \mathbf{S}$ for $f_{\mathbf{S}}$ can now be defined in the same way as its counterpart for $f_{\mathbf{B}}$.

5.3. For the candidate mating. Consider the quadratic rational function $R_{\nu}$ constructed in Theorem4.5. Denote the Fatou set and the Julia set for $R_{\nu}$ by $F\left(R_{\nu}\right)$ and $J\left(R_{\nu}\right)$ respectively. A connected component of $F\left(R_{\nu}\right)$ is called a bubble.

The critical points for $R_{\nu}$ are $\infty$ and 1. Recall that $\left\{\infty, R_{\nu}(\infty)\right\}$ is a superattracting 2-periodic orbit, and thus is contained in $F\left(R_{\nu}\right)$. Let $\mathcal{B}_{\infty}$ be the bubble 


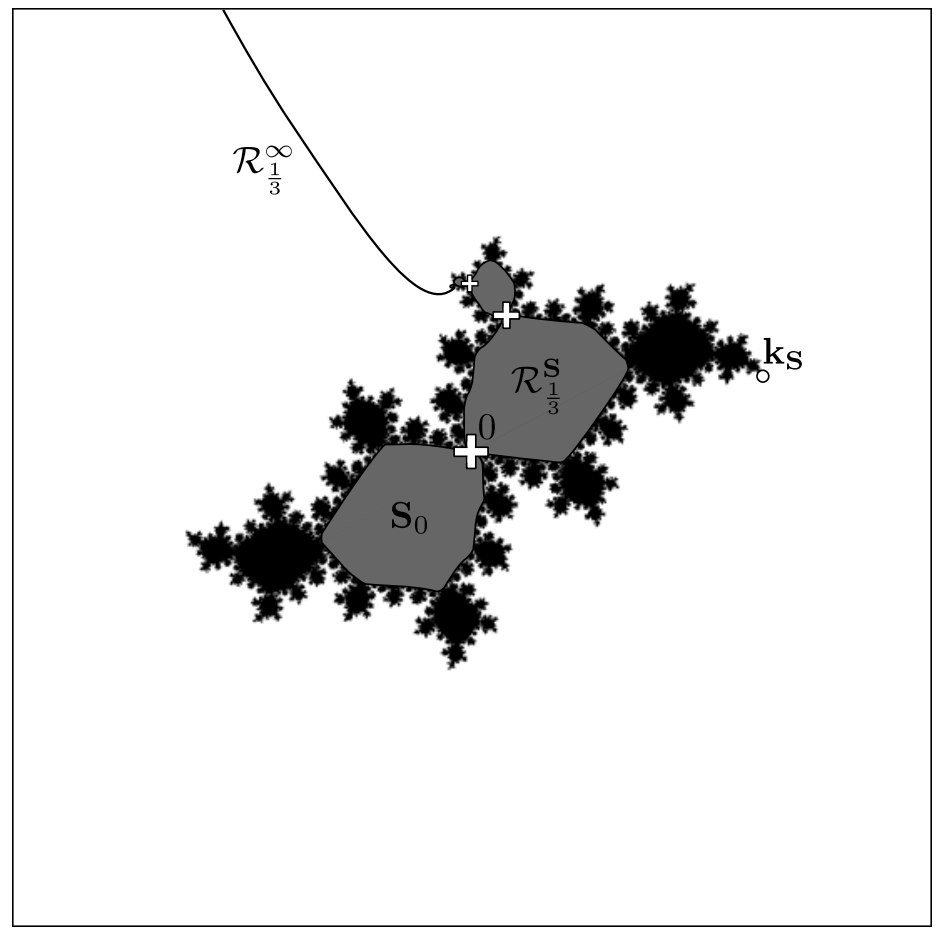

FIgURE 7. The infinite bubble ray $\mathcal{R}_{\frac{1}{3}}^{\mathrm{S}}$ for the Siegel polynomial $f_{\mathbf{S}}$. The bubbles contained in $\mathcal{R}_{\frac{1}{3}}^{\mathbf{S}}$ are colored in dark gray. The white crosses represent the set of attachment points for $\mathcal{R}_{\frac{1}{3}}^{\mathbf{S}}$.

containing $\infty$. The set

$$
\mathcal{B}:=\bigcup_{n=0}^{\infty} R_{\nu}^{-n}\left(\mathcal{B}_{\infty}\right)
$$

is the basin of attraction for $\left\{\infty, R_{\nu}(\infty)\right\}$.

The quadratic rational function $R_{\nu}$ has a Siegel fixed point at 0 with rotation number $\nu$. Denote the Siegel disc for $R_{\nu}$ (the set $\phi(\mathbb{D})$ in Section 4) by $\mathcal{S}_{0}$. As noted in the proof of Theorem A, the critical point 1 is contained in $\partial \mathcal{S}_{0}$. Consider the set of iterated preimages of $\mathcal{S}_{0}$

$$
\mathcal{S}:=\bigcup_{n=0}^{\infty} R_{\nu}^{-n}\left(\mathcal{S}_{0}\right),
$$

It is easy to see that $F\left(R_{\nu}\right)=\mathcal{B} \cup \mathcal{S}$.

Proposition 5.10. Suppose $U \subset F\left(R_{\nu}\right)$ is a bubble. Then $\partial U$ is locally connected. Proof. The result follows immediately from Proposition 2.2 and Theorem A.

Lemma 5.11. Suppose $X \subset J\left(R_{\nu}\right)$ is a closed, connected, non-recurring set (that is, $R_{\nu}^{n}(X) \cap X=\varnothing$ for all $\left.n \in \mathbb{N}\right)$. Then $X$ cannot intersect the boundary of bubbles from both $\mathcal{B}$ and $\mathcal{S}$. 
Proof. Suppose that there exists two bubbles $B \subset \mathcal{B}$ and $S \subset \mathcal{S}$ such that $X$ intersects both $\partial B$ and $\partial S$. Without loss of generality, we may assume that $B=\mathcal{B}_{\infty}$ and $S=\mathcal{S}_{0}$. Observe that $R_{\nu}^{2 n}(X)$ intersects $\partial \mathcal{B}_{\infty}$ and $\partial \mathcal{S}_{0}$ for all $n \geq 0$. Likewise, $R_{\nu}^{2 n+1}(X)$ intersects $R_{\nu}\left(\mathcal{B}_{\infty}\right)$ and $\partial \mathcal{S}_{0}$ for all $n \geq 0$.

Let $Y:=X \cup R_{\nu}^{2}(X)$, and consider the set

$$
W:=\hat{\mathbb{C}} \backslash\left(\mathcal{B}_{\infty} \cup \mathcal{S}_{0} \cup Y\right)
$$

We claim that if $C$ is a component of $W$, then $C$ is arcwise connected. Let $c$ be a point in $C \backslash \stackrel{\circ}{C} \subset \partial \mathcal{B}_{\infty} \cup \partial \mathcal{S}_{0}$. Since $Y$ is a closed set, there exists a neighborhood $N$ of $c$ such that $N \cap Y=\varnothing$. By Proposition 5.10, it follows that $c$ is arcwise

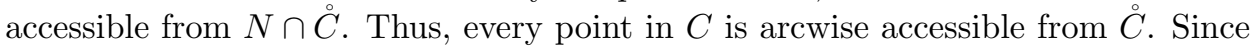
$C$ is connected, this implies that $C$ is arcwise connected.

Now, let $C^{\prime}$ be the component of $W$ that contains $R_{\nu}\left(\mathcal{B}_{\infty}\right)$. We claim that $\partial \mathcal{S}_{0} \cap W$ is not contained in $C^{\prime}$. Choose a point $x_{0}$ contained in $X \cap \partial \mathcal{S}_{0}$. Since $\partial \mathcal{S}_{0}$ is homeomorphic to a circle, we see that $\partial \mathcal{S}_{0} \backslash\left\{x_{0}, R_{\nu}^{2}\left(x_{0}\right)\right\}$ has exactly two components: $\gamma_{1}$ and $\gamma_{2}$. Choose two points $w_{1} \in \gamma_{1} \cap W$ and $w_{2} \in \gamma_{2} \cap W$. If $\partial \mathcal{S}_{0} \cap W$ is contained in $C^{\prime}$, then there exists a simple curve $\Gamma \subset C^{\prime}$ whose endpoints are $w_{1}$ and $w_{2}$. The complement of $\mathcal{S}_{0} \cup \Gamma$ has exactly two components: one which contains $x_{0}$, and one which contains $R_{\nu}^{2}\left(x_{0}\right)$. This contradicts the fact that $\mathcal{B}_{\infty} \cup Y$ is connected.

We conclude that there exists at least one connected component of $W$ that intersects $\partial \mathcal{S}_{0}$ but does not intersect $\overline{R_{\nu}\left(\mathcal{B}_{\infty}\right)}$. Denote this component by $D$. Since $X$ is non-recurring, we have

$$
R_{\nu}^{2 n+1}(X) \cap D=\varnothing \quad \text { for all } \quad n \geq 0 .
$$

However, since the orbit of $R_{\nu}\left(x_{0}\right)$ under $R_{\nu}^{2}$ is dense in $\partial \mathcal{S}_{0}$, there exists $N \geq 0$ such that

This is a contradiction.

$$
R_{\nu}^{2 N+1}\left(x_{0}\right) \in \partial \mathcal{S}_{0} \cap D
$$

Proposition 5.12. Let $B \subset \mathcal{B}$ and $S \subset \mathcal{S}$ be two bubbles. Then $\partial B \cap \partial S=\varnothing$.

Proof. Suppose that $\partial B \cap \partial S$ contains a point $x_{0}$. Since $S$ is an iterated preimage of a Siegel disc, $x_{0}$ must be non-recurrent. This contradicts Lemma 5.11 .

Proposition 5.13. There exists a unique repelling fixed point $\beta$ contained in $\partial \mathcal{B}_{\infty}$.

Proposition 5.14. Let $u$ be an iterated preimage of $\beta$ (resp. of 1 ). Then there are exactly two bubbles $U_{1}$ and $U_{2}$ in $\mathcal{B}$ (resp. in $\left.\mathcal{S}\right)$ which contain $u$ in their closure. Moreover, we have

$$
\overline{U_{1}} \cap \overline{U_{2}}=\{u\} .
$$

A bubble ray for $R_{\nu}$ can be constructed using bubbles in either $\mathcal{B}$ or $\mathcal{S}$. In the former case, the bubble ray is denoted $\mathcal{R}^{\mathcal{B}}$, and in the latter case, it is denoted $\mathcal{R}^{\mathcal{S}}$. The details of the construction will be omitted as it is very similar to the construction of a bubble ray $\mathcal{R}^{\mathrm{B}}$ for $f_{\mathbf{B}}$ or $\mathcal{R}^{\mathrm{S}}$ for $f_{\mathbf{S}}$.

Proposition 5.15. If $B \subset \mathcal{B}$ is a bubble, then there exists a unique finite bubble ray $\mathcal{R}^{\mathcal{B}} \sim\left\{B_{i}\right\}_{i=0}^{n}$ such that $B_{n}=B$. Consequently, if $\mathcal{R}_{1}^{\mathcal{B}} \sim\left\{B_{i}^{1}\right\}_{i=0}^{n}$ and $\mathcal{R}_{2}^{\mathcal{B}} \sim$ $\left\{B_{i}^{2}\right\}_{i=0}^{m}$ are two bubble rays, then there exists $N \geq 0$ such that $B_{i}^{1}=B_{i}^{2}$ for all $i \leq N$, and $B_{i}^{1} \neq B_{i}^{2}$ for all $i>N$. The analogous statement is also true for bubble rays in $\mathcal{S}$. 


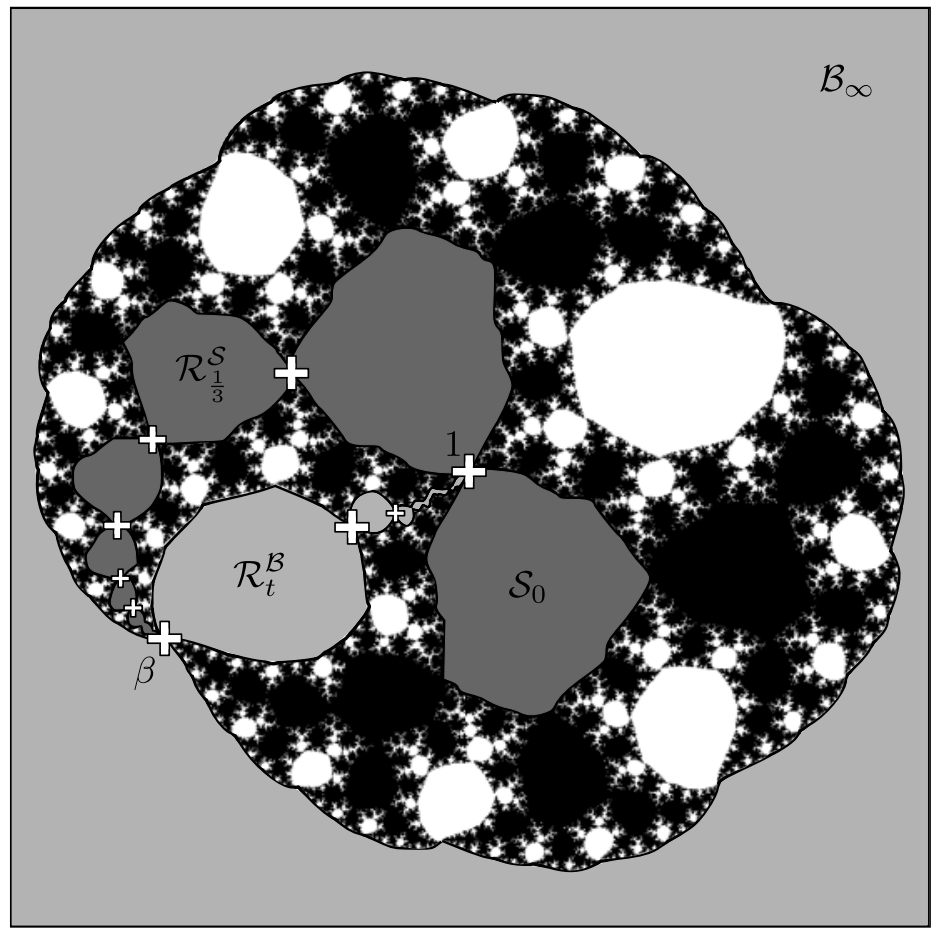

FIGURE 8. The infinite bubble rays $\mathcal{R}_{t}^{\mathcal{B}}$ with $t \approx 0.354841$ and $\mathcal{R}_{\frac{1}{3}}^{\mathcal{S}}$ for $R_{\nu}$. The bubbles contained in $\mathcal{R}_{t}^{\mathcal{B}}$ and $\mathcal{R}_{\frac{1}{3}}^{\mathcal{S}}$ are colored in light gray and dark gray respectively. The white crosses represent the set of attachment points for $\mathcal{R}_{t}^{\mathcal{B}}$ and $\mathcal{R}_{\frac{1}{3}}^{\mathcal{S}}$. Compare with Figures 6 and 7.

The bubble address of a bubble $U \subset F\left(R_{\nu}\right)$ for $R_{\nu}$ is defined in the same way as its counterpart for $f_{\mathbf{B}}$ or $f_{\mathbf{S}}$. However, since $R_{\nu}$ is not a polynomial, the external angle of a bubble ray $\mathcal{R}^{\mathcal{B}}$ or $\mathcal{R}^{\mathcal{S}}$ cannot be defined using external rays. To circumvent this problem, we need the following theorem.

Theorem 5.16. There exists a unique conformal map $\Phi_{\mathbf{B}}: \mathbf{B} \rightarrow \mathcal{B}$ such that the bubble addresses are preserved, and the following diagram commutes:

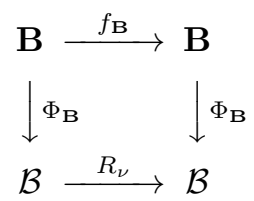

Likewise, there exists a unique conformal map $\Phi_{\mathbf{S}}: \mathbf{S} \rightarrow \mathcal{S}$ such that the bubble addresses are preserved, and the following diagram commutes:

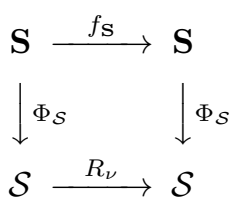


Furthermore, if $B \subset \mathbf{B}$ (resp. $S \subset \mathbf{S}$ ) is a bubble, then $\Phi_{\mathbf{B}}$ (resp. $\Phi_{\mathbf{S}}$ ) extends to a homeomorphism between $\bar{B}$ and $\overline{\Phi_{\mathbf{B}}(B)}$ (resp. $\bar{S}$ and $\overline{\Phi_{\mathbf{S}}(S)}$ ).

Proof. For each bubble $B \subset \mathbf{B}$, there exists a unique bubble $B^{\prime} \subset \mathcal{B}$ such that

$$
\operatorname{adr}(B)=\operatorname{adr}\left(B^{\prime}\right) \text {. }
$$

Define $\left.\Phi_{\mathbf{B}}\right|_{B}$ to be the unique conformal map between $B$ and $B^{\prime}$ which sends the center and the root of $B$ to the center and the root of $B^{\prime}$ respectively. Then by construction, $\Phi_{\mathbf{B}}$ conjugates $f_{\mathbf{B}}$ on $\mathbf{B}$ with $R_{\nu}$ on $\mathcal{B}$. Moreover, $\Phi_{\mathbf{B}}$ extends continuously to boundary of bubbles by Proposition 5.10 .

The map $\Phi_{\mathbf{S}}$ is defined similarly.

Let $\mathcal{R}^{\mathcal{B}} \sim\left\{B_{i}\right\}_{i=0}^{\infty}$ be an infinite bubble ray for $R_{\nu}$. The external angle of $\mathcal{R}^{\mathcal{B}}$ is defined to be the external angle of the infinite bubble ray $\mathcal{R}^{\mathbf{B}} \sim\left\{\Phi_{\mathbf{B}}^{-1}\left(B_{i}\right)\right\}_{i=0}^{\infty}$ for $f_{\mathbf{B}}$. The external angle of an infinite bubble ray $\mathcal{R}^{\mathcal{S}}$ is defined similarly. Henceforth, the infinite bubble rays for $R_{\nu}$ with external angle $t$ will be denoted by $\mathcal{R}_{t}^{\mathcal{B}}$ and $\mathcal{R}_{t}^{\mathcal{S}}$.

\section{The COnstruction of PUZzle PARTitions}

6.1. For the basilica polynomial. Consider the basilica polynomial $f_{\mathbf{B}}$ discussed in Section 5.1. By definition, the infinite bubble ray $\mathcal{R}_{t}^{\mathbf{B}}$ for $f_{\mathbf{B}}$ with external angle $t \in \mathbb{R} / \mathbb{Z}$ has the same landing point as the external ray $\mathcal{R}_{-t}^{\infty}$.

Lemma 6.1. Let $\mathcal{R}_{t_{1}}^{\mathbf{B}}$ and $\mathcal{R}_{t_{2}}^{\mathrm{B}}$ be two distinct infinite bubble rays for $f_{\mathbf{B}}$, and define

$$
X_{t_{1}, t_{2}}^{\mathrm{B}}:=\overline{\mathcal{R}_{t_{1}}^{\mathrm{B}} \cup \mathcal{R}_{-t_{1}}^{\infty}} \cup \overline{\mathcal{R}_{t_{2}}^{\mathrm{B}} \cup \mathcal{R}_{-t_{2}}^{\infty}} .
$$

Then $\hat{\mathbb{C}} \backslash X_{t_{1}, t_{2}}^{\mathrm{B}}$ has exactly two connected components: $C_{\left(t_{1}, t_{2}\right)}^{\mathbf{B}}$ and $C_{\left(t_{2}, t_{1}\right)}^{\mathrm{B}}$. If $t \in\left(t_{1}, t_{2}\right) \subset \mathbb{R} / \mathbb{Z}$, then $\mathcal{R}_{-t}^{\infty} \subset C_{\left(t_{1}, t_{2}\right)}^{\mathrm{B}}$. Similarly, if $t \in\left(t_{2}, t_{1}\right) \subset \mathbb{R} / \mathbb{Z}$, then $\mathcal{R}_{-t}^{\infty} \subset C_{\left(t_{2}, t_{1}\right)}^{\mathbf{B}}$.

Proof. First, consider the set

$$
\tilde{X}_{t_{1}, t_{2}}^{\mathrm{B}}:=K_{\mathbf{B}} \cup \overline{\mathcal{R}_{-t_{1}}^{\infty}} \cup \overline{\mathcal{R}_{-t_{2}}^{\infty}} .
$$

Observe that the complement $\hat{\mathbb{C}} \backslash \tilde{X}_{t_{1}, t_{2}}^{\mathrm{B}}$ has exactly two connected components: $\tilde{C}_{\left(t_{1}, t_{2}\right)}^{\mathrm{B}}$ and $\tilde{C}_{\left(t_{2}, t_{1}\right)}^{\mathrm{B}}$, which are given by

$$
\tilde{C}_{\left(t_{1}, t_{2}\right)}^{\mathrm{B}}=\bigcup_{t \in\left(t_{1}, t_{2}\right)} \mathcal{R}_{-t}^{\infty}
$$

and

$$
\tilde{C}_{\left(t_{2}, t_{1}\right)}^{\mathrm{B}}=\bigcup_{t \in\left(t_{2}, t_{1}\right)} \mathcal{R}_{-t}^{\infty} .
$$

Now, let $\mathcal{R}_{t_{1}}^{\mathrm{B}} \sim\left\{B_{i}^{1}\right\}_{i=0}^{\infty}$ and $\mathcal{R}_{t_{2}}^{\mathrm{B}} \sim\left\{B_{i}^{2}\right\}_{i=0}^{\infty}$, and let $N \geq 0$ be the number given in Proposition 5.4. Define

$$
\hat{X}_{t_{1}, t_{2}}^{\mathrm{B}}:=\bigcup_{i=N}^{\infty} \overline{B_{i}^{1}} \cup \bigcup_{i=N}^{\infty} \overline{B_{i}^{2}} \cup \overline{\mathcal{R}_{-t_{1}}^{\infty}} \cup \overline{\mathcal{R}_{-t_{2}}^{\infty}} .
$$

Observe that $\hat{X}_{t_{1}, t_{2}}^{\mathrm{B}} \subset \tilde{X}_{t_{1}, t_{2}}^{\mathrm{B}}$, and that the complement $\hat{\mathbb{C}} \backslash \hat{X}_{t_{1}, t_{2}}^{\mathrm{B}}$ also has exactly two connected components. Let $\hat{C}_{\left(t_{1}, t_{2}\right)}^{\mathrm{B}}$ be the component containing $\tilde{C}_{\left(t_{1}, t_{2}\right)}^{\mathrm{B}}$, and let $\hat{C}_{\left(t_{2}, t_{1}\right)}^{\mathrm{B}}$ be the component containing $\tilde{C}_{\left(t_{2}, t_{1}\right)}^{\mathrm{B}}$. 
Let $b$ be the root of the bubble $B_{N}^{1}$, and consider the set

$$
Y:=X_{t_{1}, t_{2}}^{\mathbf{B}} \backslash \hat{X}_{t_{1}, t_{2}}^{\mathbf{B}} .
$$

If $Y=\varnothing$, then the result is proved. Otherwise, there are three possibilities:

i) $Y=\overline{f_{\mathbf{B}}\left(\mathbf{B}_{0}\right)} \backslash\{b\}$,

ii) $Y=\overline{\mathbf{B}_{0}} \backslash\{b\}$, or

iii) $Y=\overline{f_{\mathbf{B}}\left(\mathbf{B}_{0}\right)} \cup\left(\bigcup_{i=0}^{N-1} \overline{B_{i}^{1}}\right) \backslash\{b\}$.

In all three cases, it follows from Proposition 5.4 that $Y$ is disjoint from either $\hat{C}_{\left(t_{1}, t_{2}\right)}^{\mathbf{B}}$ or $\hat{C}_{\left(t_{2}, t_{1}\right)}^{\mathbf{B}}$. Assume for concreteness that it is disjoint from the former. Then immediately we have $C_{\left(t_{1}, t_{2}\right)}^{\mathbf{B}} \equiv \hat{C}_{\left(t_{1}, t_{2}\right)}^{\mathbf{B}}$. Moreover, since $Y$ is simply connected, and its closure intersects $\partial \hat{C}_{\left(t_{2}, t_{1}\right)}^{\mathbf{B}}$ at only one point (namely, at $b$ ), the set $C_{\left(t_{2}, t_{1}\right)}^{\mathrm{B}}=$ $\hat{C}_{\left(t_{2}, t_{1}\right)}^{\mathrm{B}} \backslash Y$ must be connected.

The infinite bubble ray $\mathcal{R}_{0}^{\mathrm{B}}$ and the external ray $\mathcal{R}_{0}^{\infty}$ land at the same repelling fixed point $\mathbf{k}_{\mathbf{B}} \in \mathbb{C}$. For $n \in \mathbb{N}$, the puzzle partition of level $n$ for $f_{\mathbf{B}}$ is defined as

$$
\mathcal{P}_{n}^{\mathbf{B}}:=f_{\mathbf{B}}^{-n}\left(\overline{\mathcal{R}_{0}^{\mathbf{B}} \cup \mathcal{R}_{0}^{\infty}}\right)=\bigcup_{i=0}^{2^{n}-1} \overline{\mathcal{R}_{\frac{i}{2^{n}}}^{\mathbf{B}} \cup \mathcal{R}_{-\frac{i}{2^{n}}}^{\infty}} .
$$

Note that the puzzle partitions form a nested sequence: $\mathcal{P}_{1}^{\mathrm{B}} \subsetneq \mathcal{P}_{2}^{\mathrm{B}} \subsetneq \mathcal{P}_{3}^{\mathrm{B}} \ldots$

By Lemma 6.1 the complement of the puzzle partition of level $n$ is equal to

$$
\hat{\mathbb{C}} \backslash \mathcal{P}_{n}^{\mathbf{B}}=\bigsqcup_{i=0}^{2^{n}-1} C_{\left(\frac{i}{2^{n}}, \frac{i+1}{2^{n}}\right)}^{\mathbf{B}} .
$$

The puzzle piece of level $n$ for $f_{\mathbf{B}}$ is defined as

$$
P_{\left[\frac{i}{2^{n}}, \frac{i+1}{2^{n}}\right]}^{\mathbf{B}}:=\overline{C_{\left(\frac{i}{2^{n}}, \frac{i+1}{2^{n}}\right)}^{\mathbf{B}}} \quad \text { for } \quad i \in\left\{0, \ldots, 2^{n}-1\right\} .
$$

The interval $\left[\frac{i}{2^{n}}, \frac{i+1}{2^{n}}\right] \subset \mathbb{R} / \mathbb{Z}$ is referred to as the angular span of $P_{\left[\frac{i}{2^{n}}, \frac{i+1}{2^{n}}\right]}^{\mathrm{B}}$. Note that a puzzle piece of level $n \geq 2$ is mapped homeomorphically onto a puzzle piece of level $n-1$ by $f_{\mathbf{B}}$.

Proposition 6.2. Let $x \in J_{\mathbf{B}}$, and let $n \in \mathbb{N}$. If $x$ is not contained in $\partial \mathcal{P}_{n}^{\mathbf{B}}$, or there is a unique bubble $B$ contained in $\mathcal{P}_{n}^{\mathbf{B}}$ such that $x \in \partial B$, then there is a unique puzzle piece of level $n$ that contains $x$. Otherwise, $x$ is contained in exactly two puzzle pieces of level $n$.

Proof. First, suppose $x$ is not contained in $\partial \mathcal{P}_{n}^{\mathbf{B}}$. Then $x$ is contained in a single connected component of $\hat{\mathbb{C}} \backslash \mathcal{P}_{n}^{\mathbf{B}}$. The closure of this component is the unique puzzle piece of level $n$ containing $x$.

Now, suppose $x \in \partial \mathcal{P}_{n}^{\mathrm{B}}$. Then there are three possible cases:

i) There is a unique bubble $B$ contained in $\mathcal{P}_{n}^{\mathbf{B}}$ such that $x \in \partial B$.

ii) There are two bubbles $B_{1}$ and $B_{2}$ contained in $\mathcal{P}_{n}^{\mathrm{B}}$ such that $\overline{B_{1}} \cap \overline{B_{2}}=\{x\}$.

iii) The point $x$ is an iterated preimage of $\mathbf{k}_{\mathbf{B}}$.

Case i) Since $\mathcal{P}_{n}^{\mathbf{B}}$ contains finitely many bubble rays whose landing points are all distinct from $x$, we can choose a sufficiently small disc $D$ centered at $x$ such that $D \cap \mathcal{P}_{n}^{\mathbf{B}} \subset \bar{B}$. Then $D \cap\left(\hat{\mathbb{C}} \backslash \mathcal{P}_{n}^{\mathbf{B}}\right)=D \cap(\hat{\mathbb{C}} \backslash \bar{B})$ has a single connected component, which must be contained in a unique puzzle piece of level $n$. The result follows. 

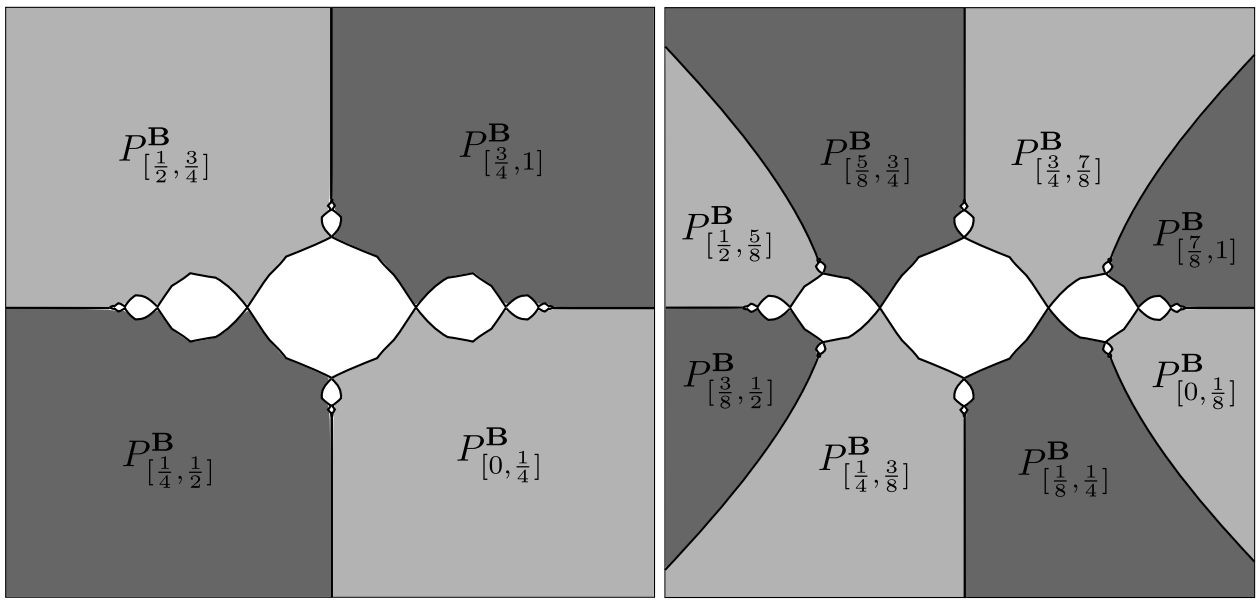

Figure 9. The puzzle pieces of level 2 (left) and 3 (right) for $f_{\mathbf{B}}$.

Case ii) By a similar reasoning as in Case i), we may choose a sufficiently small disc $D$ centered at $x$ such that $D \cap \mathcal{P}_{n}^{\mathbf{B}} \subset \overline{B_{1} \cup B_{2}}$. Thus, we see that $D \cap\left(\hat{\mathbb{C}} \backslash \mathcal{P}_{n}^{\mathbf{B}}\right)=$ $D \cap\left(\hat{\mathbb{C}} \backslash \overline{B_{1} \cup B_{2}}\right)$ has exactly two connected components, say $D_{1}$ and $D_{2}$. Let $P_{\left[t_{1}, t_{2}\right]}^{\mathrm{B}}$ be the puzzle piece of level $n$ containing $D_{1}$. Then $D_{2}$ must be contained in $C_{\left(t_{2}, t_{1}\right)}^{\mathrm{B}}$, which is disjoint from $P_{\left[t_{1}, t_{2}\right]}^{\mathrm{B}}$. This implies that $D_{2}$ is contained in a puzzle piece distinct from $P_{\left[t_{1}, t_{2}\right]}^{\mathrm{B}}$. The result follows.

Case iii) Let $t \in \mathbb{Q} / \mathbb{Z}$ be the unique dyadic rational such that the bubble ray $\mathcal{R}_{t}^{\mathbf{B}} \subset \mathcal{P}_{n}^{\mathbf{B}}$ lands at $x$. Then it is easy to see that $P_{\left[t, t+\frac{1}{2^{n}}\right]}^{\mathbf{B}}$ and $P_{\left[t-\frac{1}{2^{n}}, t\right]}^{\mathbf{B}}$ are the two puzzle pieces of level $n$ that contain $x$.

A nested puzzle sequence is a collection of puzzle pieces

$$
\Pi^{\mathbf{B}}=\left\{P_{\left[s_{k}, t_{k}\right]}^{\mathbf{B}}\right\}_{k=1}^{\infty}
$$

such that $P_{\left[s_{k+1}, t_{k+1}\right]}^{\mathrm{B}} \subsetneq P_{\left[s_{k}, t_{k}\right]}^{\mathrm{B}}$ for all $k \geq 1$. Note that this is equivalent to the condition that $\left[s_{k+1}, t_{k+1}\right] \subsetneq\left[s_{k}, t_{k}\right]$. The set

$$
L\left(\Pi^{\mathbf{B}}\right):=\bigcap_{k=1}^{\infty} P_{\left[s_{k}, t_{k}\right]}^{\mathbf{B}}
$$

is called the limit of $\Pi^{\mathrm{B}}$.

Proposition 6.3. Let $\Pi^{\mathbf{B}}=\left\{P_{\left[s_{k}, t_{k}\right]}^{\mathbf{B}}\right\}_{k=1}^{\infty}$ be a nested puzzle sequence. Then $L\left(\Pi^{\mathbf{B}}\right) \cap \mathbf{B}=\varnothing$.

Proof. Let $B \subset \mathbf{B}$ be a bubble. Since $B$ is eventually mapped to $\mathbf{B}_{0} \subset \mathcal{P}_{1}^{\mathbf{B}}$ by $f_{\mathbf{B}}$, there exists $N \geq 1$ such that $B \subset \mathcal{P}_{n}^{\mathbf{B}}$ for all $n \geq N$. This means that $B$ is disjoint from any puzzle piece of level greater than $N$. Since $P_{\left[s_{k}, t_{k}\right]}^{\mathrm{B}}$ must be of level at least $k$, we have $B \cap P_{\left[s_{k}, t_{k}\right]}^{\mathrm{B}}=\varnothing$ for all $k \geq N$. 
The external angle $t \in \mathbb{R} / \mathbb{Z}$ of $\Pi^{\mathrm{B}}$ is defined by

$$
\{t\}=\bigcap_{k=1}^{\infty}\left[s_{k}, t_{k}\right] .
$$

Henceforth, a nested puzzle sequence for $f_{\mathbf{B}}$ with external angle $t \in \mathbb{R} / \mathbb{Z}$ will be denoted by $\Pi_{t}^{\mathbf{B}}$.

Proposition 6.4. Let $\Pi_{t}^{\mathrm{B}}:=\left\{P_{\left[s_{k}, t_{k}\right]}^{\mathrm{B}}\right\}_{k=1}^{\infty}$ be a nested puzzle sequence. Then

$$
L\left(\Pi_{t}^{\mathbf{B}}\right)=\overline{\mathcal{R}_{-t}^{\infty}} \text {. }
$$

Proof. It follows from Lemma 6.1 that $\overline{\mathcal{R}_{-t}^{\infty}} \subset L\left(\Pi_{t}^{\mathbf{B}}\right)$. If $s \neq t$, then for $k$ sufficiently large, we have $s \notin\left[s_{k}, t_{k}\right]$. This means that $\overline{\mathcal{R}_{-s}^{\infty}}$ is disjoint from $P_{\left[s_{k}, t_{k}\right]}^{\mathrm{B}}$. The result now follows from Proposition 6.3.

A nested puzzle sequence $\Pi_{t}^{\mathrm{B}}$ is said to be maximal if there is no nested puzzle sequence which contains $\Pi_{t}^{\mathbf{B}}$ as a proper subset. If two nested puzzle sequences are contained in the same maximal nested puzzle sequence, they are said to be equivalent.

Proposition 6.5. Suppose $\Pi_{s}^{\mathbf{B}}$ and $\Pi_{t}^{\mathbf{B}}$ are two equivalent nested puzzle sequences. Then $s=t$, and $L\left(\Pi_{s}^{\mathbf{E}}\right)=L\left(\Pi_{t}^{\mathbf{E}}\right)$.

Proof. Let $\Pi_{s}^{\mathrm{B}}=\left\{P_{\left[s_{k}, t_{k}\right]}^{\mathrm{B}}\right\}_{k=1}^{\infty}$, and let $\hat{\Pi}_{u}^{\mathrm{B}}=\left\{P_{\left[r_{k}, u_{k}\right]}^{\mathrm{B}}\right\}_{k=1}^{\infty}$ be the maximal nested puzzle sequence containing $\Pi_{s}^{\mathrm{B}}$. Since $P_{\left[s_{k}, t_{k}\right]}^{\mathrm{B}} \subseteq P_{\left[r_{k}, u_{k}\right]}^{\mathrm{B}}$ for all $k \geq 1$, we have

$$
L\left(\Pi_{s}^{\mathbf{B}}\right) \subset L\left(\hat{\Pi}_{u}^{\mathbf{B}}\right) .
$$

On the other hand, since $\Pi_{s}^{\mathrm{B}} \subset \hat{\Pi}_{u}^{\mathrm{B}}$, we have

$$
L\left(\hat{\Pi}_{u}^{\mathbf{B}}\right) \subset L\left(\Pi_{s}^{\mathbf{B}}\right) .
$$

The proof that $s=t$ is similar.

Proposition 6.6. Let $x \in J_{\mathbf{B}}$. If $x$ is an iterated preimage of $\mathbf{b}$ or $\mathbf{k}_{\mathbf{B}}$, then there are exactly two maximal nested puzzle sequences whose limit contains $x$. Otherwise, there is a unique maximal nested puzzle sequence whose limit contains $x$.

Proof. This is an immediate consequence of Proposition 6.2

Proposition 6.7. Let $x \in J_{\mathbf{B}}$. If $x$ is an iterated preimage of $\mathbf{b}$, then $x$ is biaccessible. Otherwise, $x$ is uniaccessible.

Proof. Suppose $\Pi_{t}^{\mathbf{B}}=\left\{P_{\left[s_{k}, t_{k}\right]}^{\mathbf{B}}\right\}_{k=1}^{\infty}$ and $\tilde{\Pi}_{t}^{\mathbf{B}}=\left\{\tilde{P}_{\left[u_{k}, v_{k}\right]}^{\mathbf{B}}\right\}_{k=1}^{\infty}$ are two maximal puzzle sequences whose external angles are both equal to $t \in \mathbb{R} / \mathbb{Z}$. If $\Pi_{t}^{\mathrm{B}}$ and $\tilde{\Pi}_{t}^{\mathrm{B}}$ are nonequivalent, then there exists $k \in \mathbb{N}$ such that $\left(s_{k}, t_{k}\right) \cap\left(u_{k}, v_{k}\right)=\varnothing$. However, since $t$ is contained in both $\left[s_{k}, t_{k}\right]$ and $\left[u_{k}, v_{k}\right]$, we must have $t=t_{k}=u_{k}$ or $t=s_{k}=v_{k}$. In either case, $t$ must be a dyadic rational.

The result now follows from Propositions 6.4 and 6.6. 
6.2. For the Siegel polynomial. Consider the Siegel polynomial $f_{\mathbf{S}}$ discussed in Section 5.2. By definition, the infinite bubble ray $\mathcal{R}_{t}^{\mathbf{S}}$ for $f_{\mathbf{S}}$ with external angle $t \in \mathbb{R} / \mathbb{Z}$ has the same landing point as the external ray $\mathcal{R}_{t}^{\infty}$. The following result is a direct analog of Lemma 6.1 and can be proved in the same way.

Lemma 6.8. Let $\mathcal{R}_{t_{1}}^{\mathbf{S}}$ and $\mathcal{R}_{t_{2}}^{\mathbf{S}}$ be two infinite bubble rays for $f_{\mathbf{S}}$, and define

$$
X_{t_{1}, t_{2}}^{\mathbf{S}}:=\overline{\mathcal{R}_{t_{1}}^{\mathbf{S}} \cup \mathcal{R}_{t_{1}}^{\infty}} \cup \overline{\mathcal{R}_{t_{2}}^{\mathbf{S}} \cup \mathcal{R}_{t_{2}}^{\infty}} .
$$

Then $\hat{\mathbb{C}} \backslash X_{t_{1}, t_{2}}^{\mathbf{S}}$ has exactly two connected components: $C_{\left(t_{1}, t_{2}\right)}^{\mathbf{S}}$ and $C_{\left(t_{2}, t_{1}\right)}^{\mathbf{S}}$. If $t \in\left(t_{1}, t_{2}\right) \subset \mathbb{R} / \mathbb{Z}$, then $\mathcal{R}_{t}^{\infty} \subset C_{\left(t_{1}, t_{2}\right)}^{\mathbf{S}}$. Similarly, if $t \in\left(t_{2}, t_{1}\right) \subset \mathbb{R} / \mathbb{Z}$, then $\mathcal{R}_{t}^{\infty} \subset C_{\left(t_{2}, t_{1}\right)}^{\mathbf{S}}$.

The bubble ray $\mathcal{R}_{0}^{\mathbf{B}}$ and the external ray $\mathcal{R}_{0}^{\infty}$ both land at the same repelling fixed point $\mathbf{k}_{\mathbf{S}} \in \mathbb{C}$. A puzzle partition $\mathcal{P}_{n}^{\mathbf{S}}$, a puzzle piece $P_{\left[t_{1}, t_{2}\right]}^{\mathbf{S}}$, and a nested puzzle sequence $\Pi_{t}^{\mathbf{S}}$ for $f_{\mathbf{S}}$ are defined in the same way as their counterparts for $f_{\mathbf{B}}$.
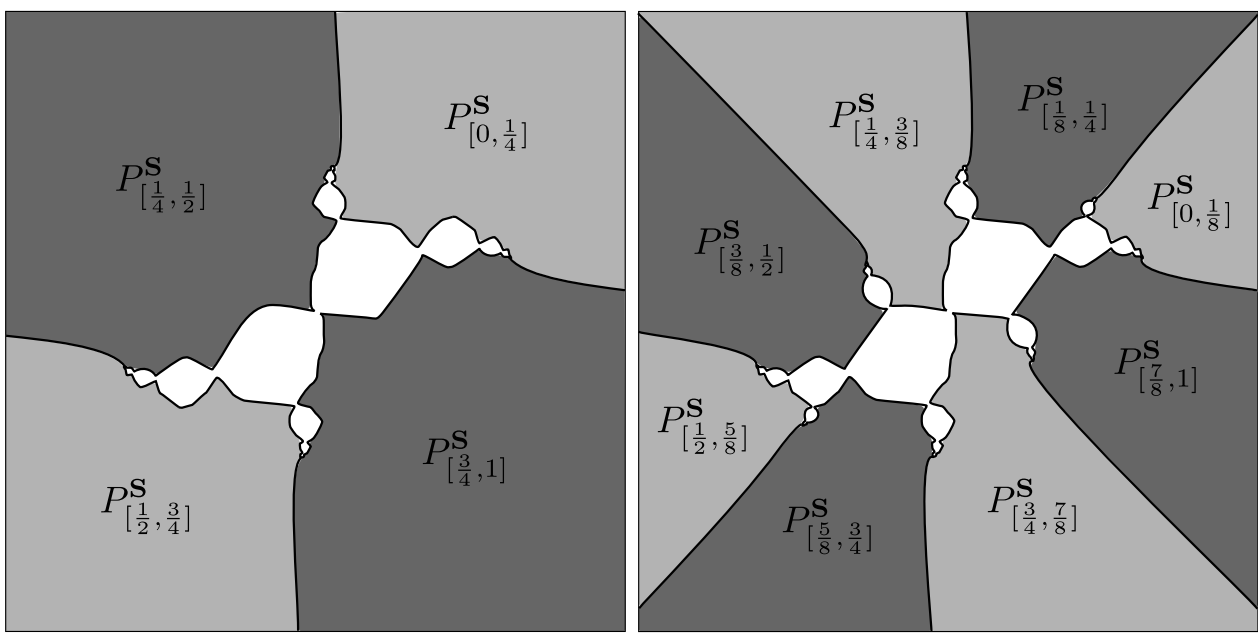

Figure 10. The puzzle pieces of level 2 (left) and 3 (right) for $f_{\mathbf{S}}$.

The following four results are analogs of Propositions 6.2, 6.4, 6.6 and 6.7. The proofs are identical, and hence, they will be omitted here.

Proposition 6.9. Let $x \in J_{\mathbf{S}}$, and let $n \in \mathbb{N}$. If $x$ is not contained in $\partial \mathcal{P}_{n}^{\mathbf{S}}$, or there is a unique bubble $S$ contained in $\mathcal{P}_{n}^{\mathbf{S}}$ such that $x \in \partial S$, then there is a unique puzzle piece of level $n$ that contains $x$. Otherwise, $x$ is contained in exactly two puzzle pieces of level $n$.

Proposition 6.10. Let $\Pi_{t}^{\mathbf{S}}:=\left\{P_{\left[s_{k}, t_{k}\right]}^{\mathbf{S}}\right\}_{k=1}^{\infty}$ be a nested puzzle sequence. Then

$$
L\left(\Pi_{t}^{\mathbf{S}}\right)=\overline{\mathcal{R}_{t}^{\infty}} .
$$

Proposition 6.11. Let $x \in J_{\mathbf{S}}$. If $x$ is an iterated preimage of 0 or $\mathbf{k}_{\mathbf{S}}$, then there are exactly two maximal nested puzzle sequences whose limit contains $x$. Otherwise, there is a unique maximal nested puzzle sequence whose limit contains $x$. 
Proposition 6.12. Let $x \in J_{\mathbf{S}}$. If $x$ is an iterated preimage of 0 , then $x$ is biaccessible. Otherwise, $x$ is uniaccessible.

6.3. For the candidate mating. Consider the quadratic rational function $R_{\nu}$ constructed in Theorem 4.5. The following result is an analog of Lemmas 6.1 and 6.8 .

Lemma 6.13. Let $\mathcal{R}_{t_{1}}^{\mathcal{B}}$ and $\mathcal{R}_{t_{2}}^{\mathcal{B}}$ be two infinite bubble rays in $\mathcal{B}$, and let $\mathcal{R}_{s_{1}}^{\mathcal{S}}$ and $\mathcal{R}_{s_{2}}^{\mathcal{S}}$ be two infinite bubble rays in $\mathcal{S}$. Suppose $\mathcal{R}_{t_{1}}^{\mathcal{B}}$ and $\mathcal{R}_{s_{1}}^{\mathcal{S}}$ land at the same point $x_{1}$, and $\mathcal{R}_{t_{2}}^{\mathcal{B}}$ and $\mathcal{R}_{s_{2}}^{\mathcal{S}}$ land at the same point $x_{2}$. Define

$$
X_{s_{1}, s_{2}}^{t_{1}, t_{2}}:=\overline{\mathcal{R}_{t_{1}}^{\mathcal{B}} \cup \mathcal{R}_{s_{1}}^{\mathcal{S}}} \cup \overline{\mathcal{R}_{t_{2}}^{\mathcal{B}} \cup \mathcal{R}_{s_{2}}^{\mathcal{S}}}
$$

Then $\hat{\mathbb{C}} \backslash X_{s_{1}, s_{2}}^{t_{1}, t_{2}}$ has exactly two connected components: $C_{\left(s_{1}, s_{2}\right)}^{\left(t_{1}, t_{2}\right)}$ and $C_{\left(s_{2}, s_{1}\right)}^{\left(t_{2}, t_{1}\right)}$, such that

$$
\begin{gathered}
\Phi_{\mathbf{B}}\left(\mathbf{B} \cap C_{\left(t_{1}, t_{2}\right)}^{\mathbf{B}}\right)=\mathcal{B} \cap C_{\left(s_{1}, s_{2}\right)}^{\left(t_{1}, t_{2}\right)}, \quad \Phi_{\mathbf{B}}\left(\mathbf{B} \cap C_{\left(t_{2}, t_{1}\right)}^{\mathbf{B}}\right)=\mathcal{B} \cap C_{\left(s_{2}, s_{1}\right)}^{\left(t_{2}, t_{1}\right)}, \\
\Phi_{\mathbf{S}}\left(\mathbf{S} \cap C_{\left(s_{1}, s_{2}\right)}^{\mathbf{S}}\right)=\mathcal{S} \cap C_{\left(s_{1}, s_{2}\right)}^{\left(t_{1}, t_{2}\right)}, \quad \text { and } \quad \Phi_{\mathbf{S}}\left(\mathbf{S} \cap C_{\left(s_{2}, s_{1}\right)}^{\mathbf{S}}\right)=\mathcal{S} \cap C_{\left(s_{2}, s_{1}\right)}^{\left(t_{2}, t_{1}\right)},
\end{gathered}
$$

where $\Phi_{\mathbf{B}}: \mathbf{B} \rightarrow \mathcal{B}$ and $\Phi_{\mathbf{S}}: \mathbf{S} \rightarrow \mathcal{S}$ are the maps given in Theorem 5.16 .

Proof. Consider the bubble rays $\mathcal{R}_{t_{1}}^{\mathbf{B}} \sim\left\{B_{i}^{1}\right\}_{i=0}^{\infty}, \mathcal{R}_{t_{2}}^{\mathrm{B}} \sim\left\{B_{i}^{2}\right\}_{i=0}^{\infty}, \mathcal{R}_{s_{1}}^{\mathbf{S}} \sim\left\{S_{i}^{1}\right\}_{i=0}^{\infty}$, and $\mathcal{R}_{s_{2}}^{\mathbf{S}} \sim\left\{S_{i}^{2}\right\}_{i=0}^{\infty}$ for $f_{\mathbf{B}}$ and $f_{\mathbf{S}}$. Let $N \geq 0$ and $M \geq 0$ be the numbers given in Propositions 5.4 and 5.8 respectively. Define

$$
Y_{t_{1}, t_{2}}^{\mathbf{B}}:=\bigcup_{i=N}^{\infty} \overline{B_{i}^{1}} \cup \bigcup_{i=N}^{\infty} \overline{B_{i}^{2}} \quad \text { and } \quad Y_{s_{1}, s_{2}}^{\mathbf{S}}:=\bigcup_{i=M}^{\infty} \overline{S_{i}^{1}} \cup \bigcup_{i=M}^{\infty} \overline{S_{i}^{2}}
$$

Recall the definition of $\hat{C}_{\left(t_{1}, t_{2}\right)}^{\mathrm{B}}$ and $\hat{C}_{\left(t_{2}, t_{1}\right)}^{\mathrm{B}}$ for $f_{\mathbf{B}}$ given in the proof of Lemma 6.1. Let $\hat{C}_{\left(s_{1}, s_{2}\right)}^{\mathbf{S}}$ and $\hat{C}_{\left(s_{2}, s_{1}\right)}^{\mathbf{S}}$ be the analogous structures for $f_{\mathbf{S}}$. Define

$$
\gamma_{\left(t_{1}, t_{2}\right)}^{\mathbf{B}}:=Y_{t_{1}, t_{2}}^{\mathbf{B}} \cap \partial \hat{C}_{\left(t_{1}, t_{2}\right)}^{\mathbf{B}} \quad \text { and } \quad \gamma_{\left(t_{2}, t_{1}\right)}^{\mathbf{B}}:=Y_{t_{1}, t_{2}}^{\mathbf{B}} \cap \partial \hat{C}_{\left(t_{2}, t_{1}\right)}^{\mathbf{B}} .
$$

The sets $\gamma_{\left(s_{1}, s_{2}\right)}^{\mathbf{S}}$ and $\gamma_{\left(s_{2}, s_{1}\right)}^{\mathbf{S}}$ are defined analogously.

The maps $\Phi_{\mathbf{B}}$ and $\Phi_{\mathbf{S}}$ extend continuously to $Y_{t_{1}, t_{2}}^{\mathbf{B}}$ and $Y_{s_{1}, s_{2}}^{\mathbf{S}}$. Define

$$
\hat{X}_{s_{1}, s_{2}}^{t_{1}, t_{2}}:=\Phi_{\mathbf{B}}\left(Y_{t_{1}, t_{2}}^{\mathbf{B}}\right) \cup \Phi_{\mathbf{S}}\left(Y_{s_{1}, s_{2}}^{\mathbf{S}}\right) \cup\left\{x_{1}, x_{2}\right\} .
$$

It follows from Propositions 5.12 and 5.15 that the complement $\hat{\mathbb{C}} \backslash \hat{X}_{s_{1}, s_{2}}^{t_{1}, t_{2}}$ has exactly two connected components. Since $\Phi_{\mathbf{B}}$ and $\Phi_{\mathbf{S}}$ are orientation preserving, the boundary of one of these components contains $\Phi_{\mathbf{B}}\left(\gamma_{\left(t_{1}, t_{2}\right)}^{\mathbf{B}}\right)$ and $\Phi_{\mathbf{S}}\left(\gamma_{\left(s_{1}, s_{2}\right)}^{\mathbf{S}}\right)$, and the boundary of the other contains $\Phi_{\mathbf{B}}\left(\gamma_{\left(t_{2}, t_{1}\right)}^{\mathbf{B}}\right)$ and $\Phi_{\mathbf{S}}\left(\gamma_{\left(s_{2}, s_{1}\right)}^{\mathbf{S}}\right)$. Denote the former component by $\hat{C}_{\left(s_{1}, s_{2}\right)}^{\left(t_{1}, t_{2}\right)}$ and the latter component by $\hat{C}_{\left(s_{2}, s_{1}\right)}^{\left(t_{2}, t_{1}\right)}$.

Now, given a bubble $U \subset \mathbf{B}$, let $\mathcal{R}_{U}^{\mathbf{B}} \sim\left\{U_{i}\right\}_{i=0}^{n}$ be the unique finite bubble ray such that $U_{n}=U$. Since $\Phi_{\mathbf{B}}$ extends to a homeomorphism on $\mathcal{R}_{U}^{\mathbf{B}} \cup \mathcal{R}_{t_{1}}^{\mathbf{B}} \cup \mathcal{R}_{t_{2}}^{\mathbf{B}}$, it follows from the above definitions together with Propositions 5.12 and 5.15 that $\Phi_{\mathbf{B}}(U) \subset \hat{C}_{\left(s_{1}, s_{2}\right)}^{\left(t_{1}, t_{2}\right)}$ if $U \subset \hat{C}_{\left(t_{1}, t_{2}\right)}^{\mathbf{B}}$, and $\Phi_{\mathbf{B}}(U) \subset \hat{C}_{\left(s_{2}, s_{1}\right)}^{\left(t_{2}, t_{1}\right)}$ if $U \subset \hat{C}_{\left(t_{2}, t_{1}\right)}^{\mathbf{B}}$. A completely symmetric argument shows that the analogous statement is true for bubbles in $\mathbf{S}$.

The rest of the proof is similar to that of Lemma 6.1 and hence, will be omitted here. 
In order to construct the puzzle partitions for $R_{\nu}$, we need to prove that every infinite periodic bubble ray lands at a repelling periodic orbit point. This requires the following classical result in holomorphic dynamics (see e.g. M1]).

Lemma 6.14 (Snail's Lemma). Let $V \subset \mathbb{C}$ be a neighborhood of 0 , and let $f: V \rightarrow$ $\mathbb{C}$ be a holomorphic function. Suppose there exists a path $\gamma:[0, \infty) \rightarrow V \backslash\{0\}$ which is mapped into itself by $f$ in such a way that $f(\gamma(t))=\gamma(t+1)$ and $\gamma$ converges to 0 . Then 0 is a fixed point for $f$, and $f^{\prime}(0)=1$ or $\left|f^{\prime}(0)\right|<1$.

Proposition 6.15. Let $\mathcal{R}_{t}=\mathcal{R}_{t}^{\mathcal{B}}$ or $\mathcal{R}_{t}^{\mathcal{S}}$ be an infinite bubble ray. If $t$ is rational, then $\mathcal{R}_{t}$ lands. If $t$ is p-periodic, then $\mathcal{R}_{t}$ lands at a repelling p-periodic point.

Proof. Let $\Omega$ be the set of cluster points for $\mathcal{R}_{t}$. Define

$$
\Lambda:=\Omega \cup\left\{\infty, R_{\nu}(\infty)\right\} \cup \overline{\mathbf{S}_{0}} .
$$

Observe that

$$
R_{\nu}^{p}: \hat{\mathbb{C}} \backslash R_{\nu}^{-p}(\Lambda) \rightarrow \hat{\mathbb{C}} \backslash \Lambda
$$

is a regular $2^{p}$-fold covering of connected hyperbolic spaces. Moreover, since $\Lambda \subsetneq$ $R_{\nu}^{-p}(\Lambda)$, the inclusion map

$$
\iota: \hat{\mathbb{C}} \backslash R_{\nu}^{-p}(\Lambda) \rightarrow \hat{\mathbb{C}} \backslash \Lambda
$$

is a strict contraction in the hyperbolic metric. Hence, the map $\iota \circ R_{\nu}^{-p}$ lifts to the universal cover $\mathbb{D}$ of $\hat{\mathbb{C}} \backslash \Lambda$ to a map

$$
\hat{R}_{\nu}^{-p}: \mathbb{D} \rightarrow \mathbb{D}
$$

which is also a strict contraction in the hyperbolic metric.

Now, choose a bubble $U \subset \mathcal{R}_{t}$ such that $\operatorname{gen}(U)>1$, and let $x_{0}$ be a point contained in $U$. For every $k \geq 0$, there exists a unique point $x_{k} \in \mathcal{R}_{t}$ such that $R_{\nu}^{k p}\left(x_{k}\right)=x_{0}$. Let $\gamma_{0} \subset \mathcal{R}_{t}$ be a curve from $x_{0}$ to $x_{1}$, and let $\gamma_{k}$ be the unique component of $R_{\nu}^{-k p}\left(\gamma_{0}\right)$ whose end points are $x_{k}$ and $x_{k+1}$.

By the strict contraction property of $\hat{R}_{\nu}^{p}$, the hyperbolic lengths of $\gamma_{n}$ must go to zero as $n$ goes to infinity. Hence, if $z \in \Omega$, then for any neighborhood $N$ of $z$, there exists a smaller neighborhood $N^{\prime} \subset N$ such that if $\gamma_{n} \cap N^{\prime} \neq \varnothing$, then $\gamma_{n} \subset N$. In other words, $R_{\nu}^{p}(N) \cap N \neq \varnothing$. Since this is true for all neighborhood of $z$, the map $R_{\nu}^{p}$ must fix $z$.

The set of fixed points for $R_{\nu}^{p}$ is discrete. Since $\Omega$ is connected, this implies that $\Omega$ must be equal to the single point set $\{z\}$. By Lemma 6.14, we conclude that $z$ is a repelling fixed point.

If $t$ is strictly preperiodic, then $\mathcal{R}_{t}$ is the preimage of some periodic infinite bubble ray. The result follows.

Proposition 6.16. The bubble rays $\mathcal{R}_{0}^{\mathcal{B}}$ and $\mathcal{R}_{0}^{\mathcal{S}}$ land at the same repelling fixed point $\kappa \in \mathbb{C}$.

Proof. The quadratic rational map $R_{\nu}$ has exactly three fixed points, two of which must be the Siegel fixed point 0 and the repelling fixed point $\beta$. Clearly, a bubble ray cannot land at 0 , so it suffices to prove that a fixed bubble ray cannot land at $\beta$.

Let $D$ be a sufficiently small disc centered at $\beta$ such that $R_{\nu}$ is conformal on $D$. The set $D \cap\left(\hat{\mathbb{C}} \backslash \overline{\mathcal{B}_{\infty} \cup R_{\nu}\left(\mathcal{B}_{\infty}\right)}\right)$ has two connected components $D_{1}$ and $D_{2}$ such that $D_{1} \subset R_{\nu}\left(D_{2}\right)$ and $D_{2} \subset R_{\nu}\left(D_{1}\right)$. Suppose $\mathcal{R}$ is a bubble ray that lands at $\beta$. Then $\mathcal{R}$ must be disjoint from either $D_{1}$ or $D_{2}$. Hence, $\mathcal{R}$ cannot be fixed. 
Proposition 6.17. Let $t \in \mathbb{R} / \mathbb{Z}$ be a dyadic rational. Then $\mathcal{R}_{t}^{\mathcal{B}}$ and $\mathcal{R}_{t}^{\mathcal{S}}$ land at the same iterated preimage of $\kappa$.

Proof. Define $D_{n}:=\left\{\frac{i}{2^{n}}\right\}_{i=0}^{2^{n}-1} \subset \mathbb{R} / \mathbb{Z}$, and let $t \in D_{n}$ for some $n \geq 0$. Note that the case $n=0$ is proved in Proposition 6.16. Proceeding inductively, assume that $n>0$, and that the result is true for the dyadic rationals in $D_{n-1}$.

If $t \in D_{n} \backslash D_{n-1}$, then $t$ can be expressed as

$$
t=\frac{i}{2^{n-1}}+\frac{1}{2^{n}} \quad \text { for some } \quad i \in\left\{0, \ldots, 2^{n-1}-1\right\} .
$$

Observe that $t$ is the unique member of $D_{n}$ contained in the interval $\left(\frac{i}{2^{n-1}}, \frac{i+1}{2^{n-1}}\right)$. It follows from Lemma 6.13 that $\mathcal{R}_{t}^{\mathcal{B}}$ is the only member of $\left\{\mathcal{R}_{s}^{\mathcal{B}}\right\}_{s \in D_{n}}$ whose landing point lies in $C_{\left(\frac{i}{2^{n}-1}, \frac{i+1}{2^{n}-1}\right)}^{\left(\frac{i}{2^{n}-1}, \frac{i+1}{2^{n}}\right)}$. Likewise, $\mathcal{R}_{t}^{\mathcal{S}}$ is the only member of $\left\{\mathcal{R}_{s}^{\mathcal{S}}\right\}_{s \in D_{n}}$ whose landing point lies in $C_{\left(\frac{i}{2^{n-1}}, \frac{i+1}{2^{n-1}}\right)}^{\left(\frac{i}{2^{n}-1}\right)}$. By Proposition 6.16, $\mathcal{R}_{t}^{\mathcal{B}}$ and $\mathcal{R}_{t}^{\mathcal{S}}$ must land at the same point.

For $n \in \mathbb{N}$, define the puzzle partition of level $n$ for $R_{\nu}$ by

$$
\mathcal{P}_{n}:=R_{\nu}^{-n}\left(\overline{\mathcal{R}_{0}^{\mathcal{B}} \cup \mathcal{R}_{0}^{\mathcal{S}}}\right)=\bigcup_{i=0}^{2^{n}-1} \overline{\mathcal{R}_{\frac{i}{2^{n}}}^{\mathcal{B}} \cup \mathcal{R}_{\frac{i}{2^{n}}}^{\mathcal{S}}} .
$$

By Lemmas 6.13 and 6.17, the complement of the puzzle partition of level $n$ is equal to

$$
\hat{\mathbb{C}} \backslash \mathcal{P}_{n}=\bigsqcup_{i=0}^{2^{n}-1} C_{\left(\frac{i}{2^{n}}, \frac{i+1}{2^{n}}\right)}^{\left(\frac{i}{2^{n}}\right)} .
$$

A puzzle piece of level $n$ for $R_{\nu}$ is defined as

$$
P_{\left[\frac{i}{2^{n}}, \frac{i+1}{2^{n}}\right]}:=\overline{C_{\left(\frac{i}{2^{n}}, \frac{i}{2^{n}}, \frac{i+1}{2^{n}}\right)}^{i n}} \quad \text { for } \quad i \in\left\{0, \ldots, 2^{n}-1\right\} .
$$

The interval $\left[\frac{i}{2^{n}}, \frac{i+1}{2^{n}}\right] \subset \mathbb{R} / \mathbb{Z}$ is referred to as the angular span of $P_{\left[\frac{i}{2^{n}}, \frac{i+1}{2^{n}}\right]}$.

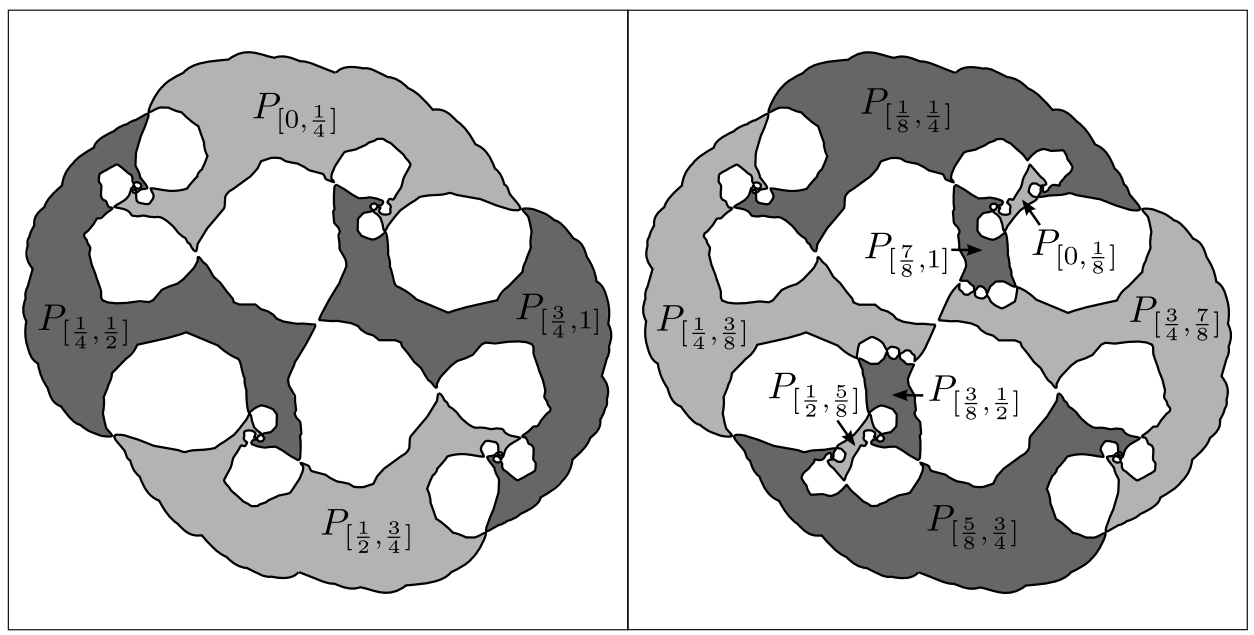

Figure 11. The puzzle pieces of level 2 (left) and 3 (right) for $R_{\nu}$. Compare with Figures 9 and 10. 
Proposition 6.18. Let $P_{\left[t_{1}, t_{2}\right]}$ be a puzzle piece with angular span $\left[t_{1}, t_{2}\right] \subset \mathbb{R} / \mathbb{Z}$. If $\mathcal{R}_{t}=\mathcal{R}_{t}^{\mathcal{B}}$ or $\mathcal{R}_{t}^{\mathcal{S}}$ is an infinite bubble ray with external angle $t \in\left[t_{1}, t_{2}\right]$, then the accumulation set of $\mathcal{R}_{t}$ is contained in $P_{\left[t_{1}, t_{2}\right]}$.

The following result is an analog of Proposition 6.2. The proof is very similar, and hence, it will be omitted here.

Proposition 6.19. Let $x \in J\left(R_{\nu}\right)$, and let $n \in \mathbb{N}$. If $x$ is not contained in $\partial \mathcal{P}_{n}$, or there is a unique bubble $U$ contained in $\mathcal{P}_{n}$ such that $x \in \partial U$, then there is a unique puzzle piece of level $n$ that contains $x$. Otherwise, $x$ is contained in exactly two puzzle pieces of level $n$.

A nested puzzle sequence $\Pi_{t}$ for $R_{\nu}$ is defined in the same way as its counterpart for $f_{\mathbf{B}}$.

Proposition 6.20. Let $x \in J\left(R_{\nu}\right)$. If $x$ is an iterated preimage of $\kappa$, $\beta$ or 1 , then there are exactly two maximal nested puzzle sequences whose limit contains $x$. Otherwise, there is exactly one maximal nested puzzle sequence whose limit contains $x$.

Proof. This is an immediate consequence of Proposition 6.19.

Proposition 6.21. Let $\Pi_{t}$ be a nested puzzle sequence for $R_{\nu}$. Its limit $L\left(\Pi_{t}\right)$ cannot intersect the boundary of bubbles from both $\mathcal{B}$ and $\mathcal{S}$.

Proof. It is easy to see that the limit set of any nested puzzle sequence is closed, connected, and contained in $J\left(R_{\nu}\right)$. Moreover, it must be either preperiodic or non-recurrent.

Now, suppose that $L\left(\Pi_{t}\right)$ intersects the boundary of bubbles from both $\mathcal{B}$ and $\mathcal{S}$. We may assume that $L\left(\Pi_{t}\right)$ contains a point $x \in \partial \mathcal{S}_{0}$. Note that the orbit of $x$ is dense in $\partial \mathcal{S}_{0}$. Hence, if $L\left(\Pi_{t}\right)$ is periodic, then $L\left(\Pi_{t}\right)$ must contain $\partial \mathcal{S}_{0}$, which is clearly impossible. Therefore, $L\left(\Pi_{t}\right)$ must be non-recurrent. This contradicts Lemma 5.11

Let $\Pi_{t}$ be a nested puzzle sequence. We say that $\Pi_{t}$ shrinks to $x$ if its limit $L\left(\Pi_{t}\right)$ is equal to $\{x\}$.

Proposition 6.22. Let $\Pi_{t}=\left\{P_{\left[s_{k}, t_{k}\right]}\right\}_{k=1}^{\infty}$ be a nested puzzle sequence, and let $\hat{\Pi}_{t}=\left\{P_{\left[r_{k}, u_{k}\right]}\right\}_{k=1}^{\infty}$ be the unique maximal nested puzzle sequence containing $\Pi_{t}$. Then $\Pi_{t}$ shrinks to a point $x \in J\left(R_{\nu}\right)$ if and only if $\hat{\Pi}_{t}$ does.

The following result is proved in the next two sections.

Theorem 6.23 (the Shrinking Theorem). Every nested puzzle sequence for $R_{\nu}$ shrinks to a point.

\section{A PRIORI BOUNDS FOR CRITICAL CIRCLE MAPS}

A $C^{2}$ homeomorphism $f: S^{1} \rightarrow S^{1}$ is called a critical circle map if it has a unique critical point $c \in S^{1}$ of cubic type. Let $\rho=\rho(f)$ be the rotation number of $f$. In this section, $f$ will be analytic, and $\rho$ will be irrational. 
The rotation number $\rho$ can be represented as an infinite continued fraction:

$$
\rho=\left[a_{1}: a_{2}: a_{3}: \ldots\right]=\frac{1}{a_{1}+\frac{1}{a_{2}+\frac{1}{a_{3}+\ldots}} .}
$$

The $n$th partial convergent of $\rho$ is the rational number

$$
\frac{p_{n}}{q_{n}}=\left[a_{1}: \ldots: a_{n}\right] .
$$

The sequence of denominators $\left\{q_{n}\right\}_{n=1}^{\infty}$ represent the closest return times of the orbit of any point to itself. It satisfies the following inductive relation:

$$
q_{n+1}=a_{n} q_{n}+q_{n-1} .
$$

Let $\Delta_{n} \subset S^{1}$ be the closed arc containing $c$ with end points at $f^{q_{n}}(c)$ and $f^{q_{n+1}}(c)$. The arc $\Delta_{n}$ can be expressed as the union of two closed subarcs $A_{n}$ and $A_{n+1}$, where $A_{n}$ has its end points at $c$ and $f^{q_{n}}(c)$. The subarc $A_{n}$ is called the $n$th critical arc. The $q_{n}$ th iterated preimage of $A_{n}$ under $f$ is denoted by $A_{-n}$. The set of closed arcs

$$
\mathcal{P}_{n}^{S^{1}}=\left\{A_{n}, f\left(A_{n}\right), \ldots, f^{q_{n+1}-1}\left(A_{n}\right)\right\} \cup\left\{A_{n+1}, f\left(A_{n+1}\right), \ldots, f^{q_{n}-1}\left(A_{n+1}\right)\right\},
$$

which are disjoint except at the end points, is a partition of $S^{1}$. The collection $\mathcal{P}_{n}^{S^{1}}$ is called the dynamical partition of level $n$. The following is an important estimate regarding dynamical partitions due to Swiątek and Herman (see $\underline{\mathrm{Sw}}]$ ):

Theorem 7.1 (Real a priori bounds). Let $f: S^{1} \rightarrow S^{1}$ be a critical circle map with an irrational rotation number $\rho$. Then for all $n$ sufficiently large, every pair of adjacent atoms in $\mathcal{P}_{n}^{S^{1}}$ have $K$-commensurate diameters for some universal constant $K>1$.

Below, we present an adaptation of complex a priori bounds of [Y1] (see also [YZ]) to our setting.

Consider the quadratic rational function $R_{\nu}$ discussed in Sections 5.3 and 6.3 . Denote the Siegel disc for $R_{\nu}$ by $\mathcal{S}_{0}$. By Theorem 4.5, there exist a Blaschke product $F_{\nu}$ and a quasiconformal map $\phi: \hat{\mathbb{C}} \rightarrow \hat{\mathbb{C}}$ such that

$$
R_{\nu}(z)=\phi \circ F_{\nu} \circ \phi^{-1}(z) \quad \text { for all } \quad z \in \hat{\mathbb{C}} \backslash \mathcal{S}_{0} .
$$

Recall that $\left\{\infty, F_{\nu}(\infty)\right\}$ and $\left\{0, F_{\nu}(0)\right\}$ are superattracting 2-periodic orbits for $F_{\nu}$. Denote the bubble (the connected component of the Fatou set) for $F_{\nu}$ containing 0 and $\infty$ by $\mathcal{A}_{0}$ and $\mathcal{A}_{\infty}$ respectively. By Theorem 4.4, the restriction of $F_{\nu}$ to $S^{1}$ is a critical circle map.

A puzzle piece of level $n$ for $F_{\nu}$ is the image of a puzzle piece of level $n$ for $R_{\nu}$ under $\phi^{-1}$. The $n$th critical puzzle piece, denoted $P_{n}^{c r i t}$, is defined inductively as follows:

(i) $P_{0}^{\text {crit }}$ is the puzzle piece of level 1 which contains the first critical arc $A_{1}$.

(ii) $P_{n}^{\text {crit }}$ is the puzzle piece which contains the preimage $\operatorname{arc} A_{-n}$, and is mapped homeomorphically onto $P_{n-1}^{\text {crit }}$ by $F_{\nu}^{q_{n}}$.

Observe that $\Pi_{\text {even }}:=\left\{P_{2 n}^{c r i t}\right\}_{n=0}^{\infty}$ and $\Pi_{\text {odd }}:=\left\{P_{2 n+1}^{c r i t}\right\}_{n=0}^{\infty}$ form two disjoint nested puzzle sequences for $F_{\nu}$ at the critical point 1 . 


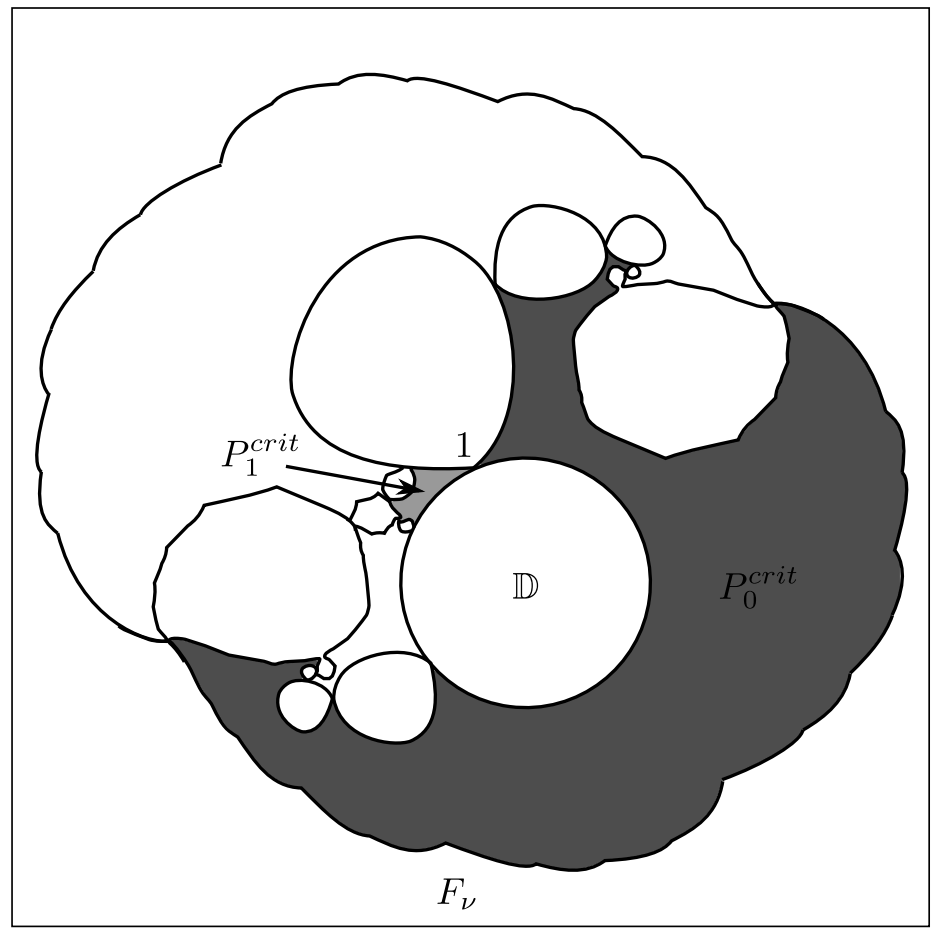

Figure 12. The 0th and 1st critical puzzle piece for $F_{\nu}$.

Lemma 7.2. Let $\mathcal{A}_{\infty} \cup F_{\nu}\left(\mathcal{A}_{\infty}\right)$ be the immediate attracting basin of the superattracting 2-periodic orbit $\left\{\infty, F_{\nu}(\infty)\right\}$ for $F_{\nu}$. Then there exists $N \geq 0$ such that for all $n \geq N$, the nth critical puzzle piece $P_{n}^{\text {crit }}$ is disjoint from the closure of $\mathcal{A}_{\infty} \cup F_{\nu}\left(\mathcal{A}_{\infty}\right)$.

Proof. The result follows immediately from Proposition 6.21.

Theorem 7.3. For all $n$ sufficiently larger than the constant $N$ in Lemma 7.2 , we have the following inequality:

$$
\frac{\operatorname{diam}\left(P_{n}^{c r i t}\right)}{\operatorname{diam}\left(A_{-n}\right)} \leq C_{1} \sqrt[3]{\frac{\operatorname{diam}\left(P_{n-1}^{c r i t}\right)}{\operatorname{diam}\left(A_{-(n-1)}\right)}}+C_{2},
$$

where $C_{1}$ and $C_{2}$ are universal constants.

Proof. Similarly to [YZ], we first lift a suitable inverse branch of $F_{\nu}$ to the universal covering space.

Define the exponential map $\operatorname{Exp}: \mathbb{C} \rightarrow \mathbb{C}$ by

$$
\operatorname{Exp}(z):=e^{2 \pi i z} .
$$

Let $I=(\tau-1, \tau) \subset \mathbb{R}$ be an open interval such that $0 \in I$, and

$$
\operatorname{Exp}(\tau)=\operatorname{Exp}(\tau-1)=F_{\nu}(1)
$$

Let

$$
\log : S^{1} \backslash\left\{F_{\nu}(1)\right\} \rightarrow I
$$


be the inverse of Exp restricted to $I$. The $n$th critical interval is defined as

$$
I_{n}:=\log \left(A_{n}\right) .
$$

Denote the component of $\operatorname{Exp}^{-1}\left(P_{n}^{c r i t}\right)$ intersecting $I$ by $\hat{P}_{n}^{c r i t}$.

Define

$$
\mathcal{A}:=\overline{\mathcal{A}_{0} \cup F_{\nu}\left(\mathcal{A}_{0}\right) \cup \mathcal{A}_{\infty} \cup F_{\nu}\left(\mathcal{A}_{\infty}\right)},
$$

and let $S \subset \mathbb{C}$ be the universal covering space of $\hat{\mathbb{C}} \backslash \mathcal{A}$ with the covering map $\left.\operatorname{Exp}\right|_{S}: S \rightarrow \hat{\mathbb{C}} \backslash \mathcal{A}$. For any given interval $J \subset \mathbb{R}$, we denote

$$
S_{J}:=(S \backslash \mathbb{R}) \cup J
$$

The restriction of the map $F_{\nu}$ to $S^{1}$ is a homeomorphism, and hence, has an inverse. We define a lift $\phi: I \rightarrow I$ of $\left(\left.F_{\nu}\right|_{\partial \mathbb{D}}\right)^{-1}$ by

$$
\phi(x):=\log \circ F_{\nu}^{-1} \circ \operatorname{Exp}(x) .
$$

Note that $\phi$ is discontinuous at $\log \left(F_{\nu}^{2}(1)\right)$, which is mapped to $\tau-1$ and $\tau$ by $\phi$. Let $n \in \mathbb{N}$. By the combinatorics of critical circle maps, the $k$ th iterate of $\phi$ on $I_{n}$ is continuous for all $1 \leq k \leq q_{n}$. By monodromy theorem, $\phi^{k}$ extends to a conformal map on $S_{I_{n}}$.

For $z \in S_{J}$, let $l_{z}$ and $r_{z}$ be the line segment connecting $z$ to $\tau-1$ and $z$ to $\tau$ respectively. The smaller of the outer angles formed between $l_{z}$ and $(-\infty, \tau-1)$, and $r_{z}$ and $(\tau,+\infty)$ is denoted $\widehat{(z, J)}$.

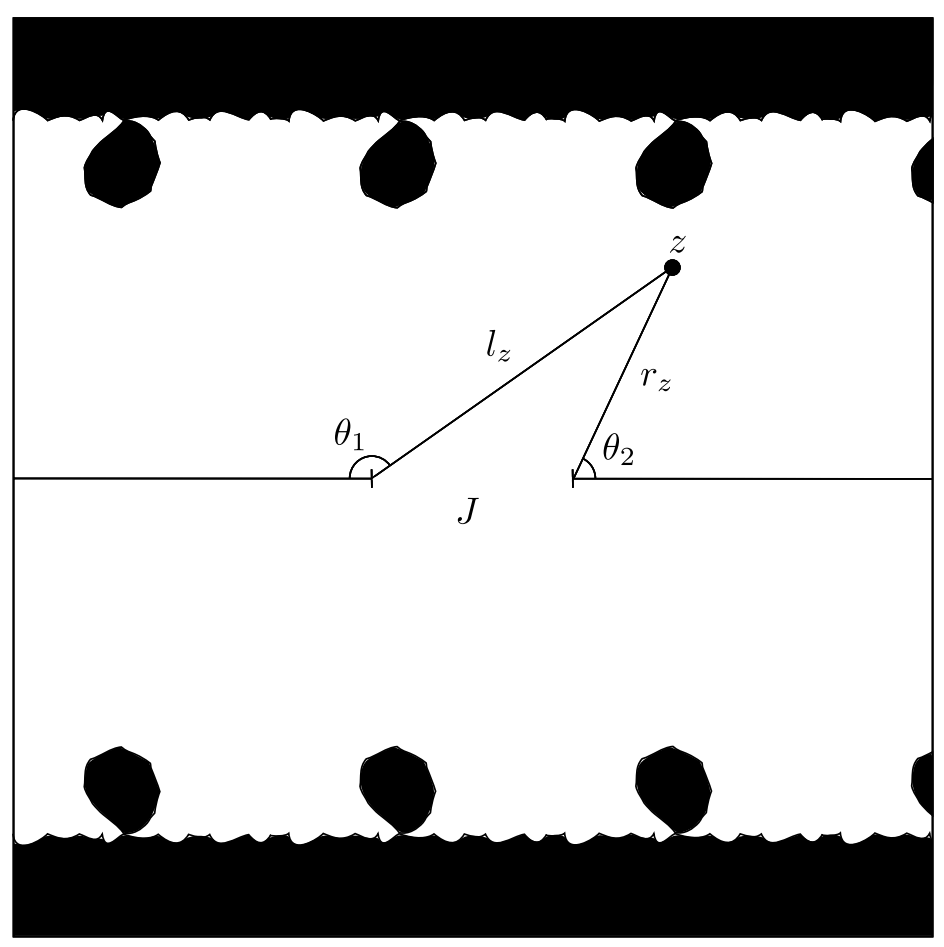

FIGURE 13. Illustration of $\widehat{(z, J)}=\min \left(\theta_{1}, \theta_{2}\right)$. 


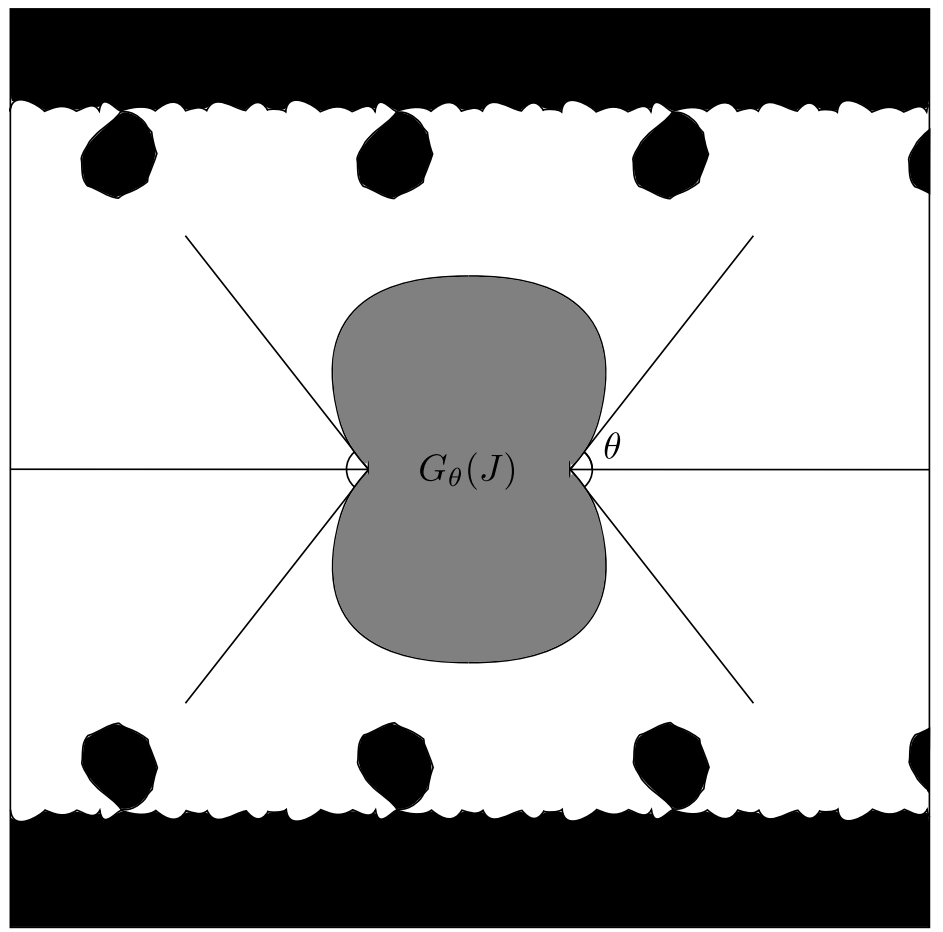

FIGURE 14. Illustration of the hyperbolic neighborhood $G_{\theta}(J)$.

Denote the hyperbolic distance in $S_{J}$ by $\operatorname{dist}_{S_{J}}$. A hyperbolic neighborhood $\{z \in$ $\left.S_{J} \mid \operatorname{dist}_{S_{J}}(z, J)\right\}$ of $J$ forms an angle $\theta \in(0, \pi)$ with $\mathbb{R}$. Denote this neighborhood by $G_{\theta}(J)$. Observe that $G_{\theta}(J) \subset\left\{z \in S_{J} \mid \widehat{(z, J)}>\theta\right\}$.

For $n \in \mathbb{N}$, define $E_{n} \subset S^{1}$ as the open arc containing 1 with end points at $F_{\nu}^{q_{n+1}}(1)$, and $F_{\nu}^{q_{n}-q_{n+1}}(1)$. Observe that $E_{n}$ contains the critical arcs $A_{n}$ and $A_{n+1}$. Define

$$
G_{\theta}^{n}:=G_{\theta}\left(\log \left(E_{n}\right)\right) .
$$

Consider the constant $N$ in Lemma 7.2 Since $P_{N}^{c r i t} \cup P_{N+1}^{c r i t}$ is disjoint from the closure of $\mathcal{A}$, it is contained in some annulus $E \Subset \hat{\mathbb{C}} \backslash \mathcal{A}$. Let $\breve{S} \Subset S$ be the universal cover of $E$ with the covering map $\left.\operatorname{Exp}\right|_{\breve{S}}$. Choose $\theta$ such that $\hat{P}_{N+2}^{c r i t} \cup \hat{P}_{N+3}^{c r i t} \subset G_{\theta}^{N+1}$. Then we have $\hat{P}_{n}^{\text {crit }} \subset G_{\theta}^{N+1}$ for all $n \geq N+3$.

Now, suppose we are given $n \geq N+3$. Let

$$
J_{0}:=I_{n}, \quad J_{-1}:=\phi\left(J_{0}\right), \quad \ldots, \quad J_{-q_{n}}:=\phi^{q_{n}}\left(I_{n}\right),
$$

be the orbit of $I_{n}$ under $\phi$. Given any point $z_{0} \in S_{J_{0}}$, let

$$
z_{0}, \quad z_{-1}:=\phi\left(z_{0}\right), \quad \ldots, \quad z_{-q_{n}}:=\phi^{q_{n}}\left(z_{0}\right),
$$

be the orbit of $z_{0}$ under $\phi$.

The following three lemmas are adaptations of Lemmas 2.1, 4.2 and 4.4 in [Y1] and Lemmas 6.1, 6.2 and 6.3 in [YZ: 
Lemma 7.4. Consider the orbit (7.2). Let $k \leq q_{n}-1$. Assume that for some $i$ between 0 and $k$, we have $z_{i} \in \breve{S}$ and $\left(\widehat{z_{-i}, J_{-i}}\right)>\epsilon$. Then

$$
\frac{\operatorname{dist}\left(z_{-k}, J_{-k}\right)}{\left|J_{-k}\right|} \leq C \frac{\operatorname{dist}\left(z_{-i}, J_{-i}\right)}{\left|J_{-i}\right|}
$$

for some constant $C=C(\epsilon, \breve{S})>0$.

Lemma 7.5. Let $J$ and $J^{\prime}$ be two consecutive returns of the orbit (17.1) of $J_{0}$ to $I_{m}$ for $1<m<n$, and let $\zeta$ and $\zeta^{\prime}$ be the corresponding points of the inverse orbit (17.2). If $\zeta \in G_{\theta}^{m}$, then either $\zeta^{\prime} \in G_{\theta}^{m}$ or $\left.\overline{\left(\zeta^{\prime}, J^{\prime}\right.}\right)>\epsilon$ and $\operatorname{dist}\left(\zeta^{\prime}, J^{\prime}\right)<C\left|I_{m}\right|$, where the constants $\epsilon$ and $C$ are independent of $m$.

Lemma 7.6. Let $J$ be the last return of the orbit (7.1) to the interval $I_{m}$ preceding the first return to $I_{m+1}$ for $1 \leq m \leq n-1$, and let $J^{\prime}$ and $J^{\prime \prime}$ be the first two returns to $I_{m+1}$. Let $\zeta, \zeta^{\prime}$ and $\zeta^{\prime \prime}$ be the corresponding points in the inverse orbit (17.2), so that $\zeta^{\prime}=\phi^{q_{m}}(\zeta)$ and $\zeta^{\prime \prime}=\phi^{q_{m+2}}\left(\zeta^{\prime}\right)$. Suppose that $\zeta \in G_{\theta}^{m}$. Then either

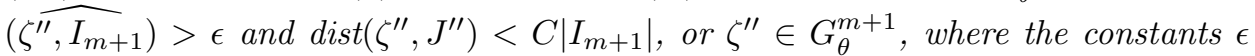
and $C$ are independent of $m$.

The interested reader can follow the proofs of Lemmas 7.4, 7.5 and 7.6, and the rest of the proof of Theorem 7.3 in [YZ mutatis mutandis.

Corollary 7.7. For all $n$ sufficiently larger than the constant $N$ in Lemma 7.2 , $\operatorname{diam}\left(P_{n}^{\text {crit }}\right)$ is $K$-commensurate to $\operatorname{diam}\left(A_{-n}\right)$ for some universal constant $K \geq 1$. Consequently, $\operatorname{diam}\left(P_{n}^{\text {crit }}\right) \rightarrow 0$ as $n \rightarrow \infty$.

Proof. It suffices to show that any sequence of positive numbers $\left\{a_{n}\right\}_{n=0}^{\infty}$ satisfying the relation

$$
a_{n} \leq C_{1} \sqrt[3]{a_{n-1}}+C_{2} \quad \text { for all } \quad n \geq 1
$$

is bounded.

Consider the sequence $\left\{b_{n}\right\}_{n=0}^{\infty}$ defined inductively by

i) $b_{0}=\max \left(1, a_{0}\right)$,

ii) $b_{n}=C \sqrt[3]{b_{n-1}}$,

where $C$ is chosen so that

$$
C \sqrt[3]{k} \geq C_{1} \sqrt[3]{k}+C_{2} \quad \text { for all } \quad k \geq 1 .
$$

It is easy to see that $b_{n} \geq a_{n}$ for all $n$.

A straightforward computation shows that

$$
b_{n}=C^{1+\frac{1}{3}+\ldots+\frac{1}{3^{n-1}} \sqrt[3]{n-1}} \sqrt{b_{0}} \stackrel{n \rightarrow \infty}{\longrightarrow} C^{\frac{3}{2}} .
$$

Hence, $\left\{b_{n}\right\}_{n=0}^{\infty}$ and therefore, $\left\{a_{n}\right\}_{n=0}^{\infty}$ are bounded.

The following result we record for later use:

Lemma 7.8. For all $n$ sufficiently large, the $n$th critical puzzle piece $P_{n}^{\text {crit }}$ contains a Euclidean disc $D_{n}$ such that $\operatorname{diam}\left(D_{n}\right)$ is $K$-commensurate to $\operatorname{diam}\left(P_{n}^{\text {crit }}\right)$ for some universal constant $K>1$. 
Proof. Let $D_{1}$ be a disc centered at 1 such that $F_{\nu}^{q_{n}}(1) \in \partial D_{1}$. The map $\left.F_{\nu}^{q_{n}}\right|_{A_{n}}$ has a well-defined inverse branch which extends to $D_{1}$. Denote this inverse branch by $\psi_{n}$. As a consequence of real a priori bounds, we have the following estimate:

$$
\frac{1}{\left|K_{1}\right|} \leq\left|\psi_{n}^{\prime}(1)\right| \leq\left|K_{1}\right|
$$

where $K_{1}$ is some universal constant independent of $n$.

Observe that the preimage of $\mathbb{D}$ under $F_{\nu}$ consists of two connected components $U_{\text {in }} \subset \mathbb{D}$ and $U_{\text {out }} \subset \mathbb{C} \backslash \overline{\mathbb{D}}$. Moreover, $\overline{U_{\text {in }}} \cap \overline{U_{\text {out }}}=\{1\}$. It is not difficult to see that $\psi_{n}$ extends to $U_{\text {out }}$, and that $\psi_{n}\left(U_{\text {out }}\right) \subset P_{n}^{\text {crit }}$.

Now, choose a subdisc $D_{2} \subset D_{1} \cap U_{\text {out }}$ such that the annulus $A=D_{1} \backslash \overline{D_{2}}$ satisfies the following estimate

$$
\frac{1}{\left|K_{2}\right|} \leq \bmod (A) \leq\left|K_{2}\right|
$$

for some universal constant $K_{2}$ independent of $n$. By Koebe distortion theorem, $\psi_{n}$ has uniformly bounded distortion on $D_{2}$. Since $\psi_{n}\left(D_{2}\right) \subset \psi_{N}\left(U_{\text {out }}\right) \subset P_{n}^{\text {crit }}$, the result follows.

\section{THE PROOF OF THE SHRINKING THEOREM}

We are ready to prove the shrinking theorem stated at the end of Section 6 The proof will be split into three propositions.

Proposition 8.1. If $\Pi_{t}$ is a nested puzzle sequence such that $L\left(\Pi_{t}\right)$ contains $\beta$ or $\kappa$, then $\Pi_{t}$ shrink to a point.

Proof. We prove the result in the case where $L\left(\Pi_{t}\right)$ contains $\kappa$. The proof of the other case is similar.

Since $L\left(\Pi_{t}\right)$ contains $\kappa$, it follows that $t=0$. Observe that $L\left(\Pi_{0}\right)$ is invariant under $R_{\nu}$. Hence, $L\left(\Pi_{0}\right) \cap \partial \mathcal{S}_{0}=\varnothing$.

Let $D_{r}$ be a disc of radius $r>0$ centered at $\kappa$. Since $\kappa$ is a repelling fixed point, if $r$ is sufficiently small, then $D_{r}$ is mapped into itself by an appropriate inverse branch of $R_{\nu}$. This inverse branch extends to a map $g: N \rightarrow N$, where $N$ is a neighborhood of $L\left(\Pi_{0}\right)$ which is disjoint from $\partial \mathcal{S}_{0}$ and therefore, the closure of the post critical set for $R_{\nu}$.

Any set compactly contained within $N$ converges to $\kappa$ under iteration of $R_{\nu}$. It follows that $L\left(\Pi_{0}\right)=\{\kappa\}$.

For the proof of the remaining two propositions, it will be more convenient for us to work with the Blaschke product $F_{\nu}$ rather than $R_{\nu}$ itself. It is clear from the definition that a nested puzzle sequence for $R_{\nu}$ shrinks if and only if the corresponding nested puzzle sequence for $F_{\nu}$ shrinks.

Proposition 8.2. If $\Pi_{t}$ is a nested puzzle sequence such that $1 \in L\left(\Pi_{t}\right)$, then $\Pi_{t}$ shrink to 1

Proof. Recall the definition of critical puzzle pieces $\left\{P_{n}^{c r i t}\right\}_{n=0}^{\infty}$ for $F_{\nu}$ in Section 7 . Let $\hat{\Pi}_{\text {even }}$ and $\hat{\Pi}_{\text {odd }}$ be the maximal nested puzzle sequence containing $\left\{P_{2 n}^{\text {crit }}\right\}_{n=0}^{\infty}$ and $\left\{P_{2 n+1}^{c r i t}\right\}_{n=0}^{\infty}$ respectively. Corollary 7.7 and Proposition 6.22 imply that $\hat{\Pi}_{\text {even }}$ and $\hat{\Pi}_{\text {odd }}$ both shrink to 1 . By Proposition 6.20 , there is no other maximal nested puzzle sequence at 1 . 
For the proof of the final proposition, we need the following lemma.

Lemma 8.3. Let $f: \hat{\mathbb{C}} \rightarrow \mathbb{C}$ be a rational map of degree $d>1$. Let $\left\{\left(\left.f\right|_{U}\right)^{-n}\right\}_{n=0}^{\infty}$ be a family of univalent inverse branches of $f$ restricted to a domain $U$. Suppose $U \cap J(f) \neq \varnothing$. If $V \Subset U$, then

$$
\operatorname{diam}\left(\left(\left.f\right|_{U}\right)^{-n}(V)\right) \rightarrow 0
$$

as $n \rightarrow \infty$.

Proposition 8.4. Let $w_{0}$ be a point in the Julia set $J\left(R_{\nu}\right)$ which is not an iterated preimage of $\kappa, \beta$ or 1 . If $\Pi_{t}$ is a nested puzzle sequence such that $w_{0} \in L\left(\Pi_{t}\right)$, then $\Pi_{t}$ shrinks to $w_{0}$.

Proof. Let $z_{0}:=\phi^{-1}\left(w_{0}\right)$, and consider the forward orbit

$$
\mathcal{O}=\left\{z_{n}\right\}_{n=0}^{\infty}
$$

of $z_{0}$ under $F_{\nu}$. The proof splits into two cases.

Case 1. Suppose there exists some critical puzzle piece $P_{M}^{c r i t}$ such that

$$
\mathcal{O} \cap P_{M}^{c r i t}=\varnothing \text {. }
$$

Let $z_{\infty}$ be an accumulation point of $\mathcal{O}$, and let $P^{\infty}$ be the puzzle piece of level $M$ containing $z_{\infty}$. Observe that the orbit of the critical point 1 is dense in $\partial \mathbb{D}$. Hence, $P^{\infty}$ must be disjoint from $\partial \mathbb{D}$, since otherwise, $P^{\infty}$ would map into $P_{M}^{c r i t}$ by some appropriate inverse branch of $F_{\nu}$.

Let $U \subset \mathbb{C} \backslash \overline{\mathbb{D}}$ be a neighborhood of $P^{\infty}$, and choose a subsequence of orbit points $\left\{z_{n_{k}}\right\}_{k=0}^{\infty}$ from $\mathcal{O}$ such that $z_{n_{k}} \in P^{\infty}$. For each $k$, let

$$
g_{k}: U \rightarrow \mathbb{C}
$$

be the inverse branch of $F_{\nu}^{n_{k}}$ that maps $z_{n_{k}}$ to $z_{0}$. Since $P^{\infty}$ intersects the Julia set for $F_{\nu}$, the nested puzzle sequence

$$
\Pi:=\left\{g_{k}\left(P^{\infty}\right)\right\}_{k=0}^{\infty}
$$

must shrink to $z_{0}$ by Lemma 8.3 .

Case 2. Suppose the critical point 1 is an accumulation point of $\mathcal{O}$. Then there exists an increasing sequence of numbers $\left\{n_{k}\right\}_{k=0}^{\infty}$ such that

$$
\mathcal{O} \cap P_{n_{k}}^{c r i t} \neq \varnothing \text {. }
$$

Fix $k$, and let $z_{m_{k}}$ be the first orbit point that enters the critical puzzle piece $P_{n_{k}}^{\text {crit }}$. Let

$$
P^{-n} \subset F_{\nu}^{-n}\left(P_{n_{k}}^{c r i t}\right)
$$

be the $n$th pullback of $P_{n_{k}}^{c r i t}$ along the orbit

$$
z_{0} \mapsto z_{1} \mapsto \ldots \mapsto z_{m_{k}}
$$

Suppose that $P^{-n}$ intersects 1 for some $n>0$. Then for all $m \leq n$, the puzzle piece $P^{-m}$ must intersect $\partial \mathbb{D}$. Recall that $P_{n_{k}}^{c r i t}$ contains the the preimage arc $A_{-n_{k}}$. Hence, for every $m \leq n$, the puzzle piece $P^{-m}$ contains the $m$ th preimage of $A_{-n_{k}}$ under $\left.F_{\nu}\right|_{\partial \mathbb{D}}$. By the combinatorics of critical circle maps, it follows that $P^{-q_{n_{k}}}$ must be the first puzzle piece in the backward orbit $\left\{P^{-1}, P^{-2}, \ldots, P^{-m_{k}}\right\}$ to intersect 1 . 
Since there are exactly two maximal nested puzzle sequences whose limit contains 1, all puzzle pieces of level $n>n_{k}+q_{n_{k}}$ which intersect 1 must be contained in either $P_{n_{k}}^{\text {crit }}$ or $P^{-q_{n_{k}}}$. Either case would contradict the fact that $z_{m_{k}}$ is the first orbit point to enter $P_{n_{k}}^{\text {crit }}$. Therefore, $P^{-n}$ does not intersect 1 for all $n \geq q_{n_{k}}$.

Let $m \leq m_{k}$ be the last moment when the backward orbit of $P^{0}=P_{n_{k}}^{c r i t}$ intersect $\partial \mathbb{D}$. By Theorem 7.1. Corollary 7.7 and combinatorics of critical circle maps, the distance between $P^{-m}$ and $F_{\nu}(1)$ is commensurate to $\operatorname{diam}\left(P^{-m}\right)$. Hence, the distance between $P^{-m-1}$ and 1 is commensurate to diam $\left(P^{-m-1}\right)$. Therefore, by Theorem 7.1 and the Koebe distortion theorem, the inverse branch of $F_{\nu}^{m_{k}}$ along the orbit (8.1) can be expressed as either

$$
\left.F_{\nu}^{-m_{k}}\right|_{P_{n_{k}}^{c r i t}}=\eta
$$

if $1 \notin P_{n}$ for all $n>0$, or

$$
\left.F_{\nu}^{-m_{k}}\right|_{P_{n_{k}}^{c r i t}}=\zeta_{1} \circ Q \circ \zeta_{2}
$$

if $1 \in P^{-q_{n_{k}}}$, where $\eta, \zeta_{1}$ and $\zeta_{2}$ are conformal maps with bounded distortion, and $Q$ is a branch of the cubic root.

Now, by Lemma 7.8, $P_{n_{k}}^{\text {crit }}$ contains a Euclidean disc $D_{n_{k}}$ such that $\operatorname{diam}\left(D_{n_{k}}\right)$ is commensurate to $\operatorname{diam}\left(P_{n_{k}}^{c r i t}\right)$. The above argument implies that the puzzle piece $P^{-m_{k}}$ must also contain a Euclidean disc $D$ such that diam $(D)$ is commensurate to $\operatorname{diam}\left(P^{-m_{k}}\right)$. Hence, $\operatorname{diam}\left(P^{-m_{k}}\right) \rightarrow 0$ as $k \rightarrow \infty$, and the nested puzzle sequence

$$
\Pi:=\left\{P^{-m_{k}}\right\}_{k=0}^{\infty}
$$

must shrink to $z_{0}$.

As an application of the shrinking theorem, we prove that every infinite bubble ray for $R_{\nu}$ lands.

Proposition 8.5. Every infinite bubble ray for $R_{\nu}$ lands.

Proof. Let $\mathcal{R}_{t}$ be an infinite bubble ray, and let $\Omega$ be its accumulation set. If $t$ is a dyadic rational, then $\mathcal{R}_{t}$ lands at an iterated preimage of $\kappa$. Otherwise, there exists a unique nested maximal puzzle sequence $\Pi_{t}=\left\{P_{\left[s_{k}, t_{k}\right]}\right\}_{k=1}^{\infty}$ with external angle equal to $t$. By Proposition [6.18, $\Omega$ must be contained in $P_{\left[s_{k}, t_{k}\right]}$ for all $k \geq 1$. The result now follows from the shrinking theorem.

\section{The proof of Conformal mateability (Theorem B)}

We are ready to prove that $R_{\nu}$ is a conformal mating of $f_{\mathbf{B}}$ and $f_{\mathbf{S}}$. Recall the maps $\Phi_{\mathbf{B}}$ and $\Phi_{\mathbf{S}}$ in Theorem 5.16 defined on the union of the closure of every bubble in $\mathbf{B}$ and $\mathbf{S}$ respectively. Our first task is to continuously extend $\Phi_{\mathbf{B}}$ and $\Phi_{\mathbf{S}}$ to the filled Julia sets $K_{\mathbf{B}}=\overline{\mathbf{B}}$ and $K_{\mathbf{S}}=\overline{\mathbf{S}}$. For brevity, we will limit our discussion to $\Phi_{\mathbf{S}}$. The map $\Phi_{\mathbf{B}}$ can be extended in a completely analogous way.

Let $\tilde{\Phi}_{\mathbf{S}}: J_{\mathbf{S}} \rightarrow J\left(R_{\nu}\right)$ be the map defined as follows. For $x \in J_{\mathbf{S}}$, let $\Pi_{t}^{\mathbf{S}}=$ $\left\{P_{\left[s_{k}, t_{k}\right]}^{\mathbf{S}}\right\}_{k=1}^{\infty}$ be a maximal nested puzzle sequence whose limit contains $x$. By the shrinking theorem, the corresponding maximal nested puzzle sequence $\Pi_{t}=$ $\left\{P_{\left[s_{k}, t_{k}\right]}\right\}_{k=1}^{\infty}$ for $R_{\nu}$ must shrink to a single point, say $y \in J\left(R_{\nu}\right)$. Define $\tilde{\Phi}_{\mathbf{S}}(x):=y$. We claim that $\tilde{\Phi}_{\mathbf{S}}$ is a continuous extension of $\Phi_{\mathbf{S}}$ on $J_{\mathbf{S}}$.

Proposition 9.1. Let $S \subset \mathbf{S}$ be a bubble. If $x \in \partial S$, then $\tilde{\Phi}_{\mathbf{S}}(x)=\Phi_{\mathbf{S}}(x)$. 
Proof. Let $z:=\Phi_{\mathbf{S}}(x)$. It is easy to see from the proof of Lemma 6.13 that $z \in$ $\overline{\hat{C}_{\left(s_{k}, t_{k}\right)}^{\left(s_{k}, t_{k}\right)}}$ for all $k \geq 1$. It follows immediately that $z \in P_{\left[s_{k}, t_{k}\right]}$ for all $k \geq 1$, and hence, $\{z\}=L\left(\Pi_{t}\right)=\{y\}$.

Proposition 9.2. The map $\tilde{\Phi}_{\mathbf{S}}: J_{\mathbf{S}} \rightarrow J\left(R_{\nu}\right)$ is well defined.

Proof. Suppose there are two maximal nested puzzle sequences at $x \in J_{\mathbf{S}}$. By Proposition 6.11, $x$ is either an iterated preimage of $\mathbf{k}_{\mathbf{S}}$ or 0 . The first case follows from Proposition 6.18. The second case follows from Proposition 9.1

Proposition 9.3. Define $\Phi_{\mathbf{S}}(x):=\tilde{\Phi}_{\mathbf{S}}(x)$ for all $x \in J_{\mathbf{S}}$. The extended map $\Phi_{\mathbf{S}}: K_{\mathbf{S}} \rightarrow \hat{\mathbb{C}}$ is continuous.

Proof. It suffices to show that if $\left\{x_{i}\right\}_{i=0}^{\infty} \subset K_{\mathbf{S}}$ is a sequence converging to $x \in J_{\mathbf{S}}$, then the sequence of image points $\left\{y_{i}=\Phi_{\mathbf{S}}\left(x_{i}\right)\right\}_{i=0}^{\infty}$ converges to $y=\Phi_{\mathbf{S}}(x)$. The proof splits into four cases:

i) The point $x$ is an iterated preimage of 0 .

ii) There exists a unique bubble $S \subset \mathbf{S}$ such that $x \in \partial S$.

iii) The point $x$ is an iterated preimage of $\mathbf{k}_{\mathbf{S}}$.

iv) Otherwise.

Case i) By Proposition 5.7 there exist exactly two bubbles $S_{1}$ and $S_{2}$ which contain $x$ in their boundary. Moreover, we have $\{x\}=\overline{S_{1}} \cap \overline{S_{2}}$. By Proposition 9.1, any subsequence of $\left\{x_{i}\right\}_{i=0}^{\infty}$ contained in $\overline{S_{1}} \cup \overline{S_{2}}$ is mapped under $\Phi_{\mathbf{S}}$ to a sequence which converges to $y$. Hence, we may assume that $x_{i}$ is not contained $\overline{S_{1}} \cup \overline{S_{2}}$ for all $i \geq 0$.

By Proposition 6.11, there are exactly two maximal nested puzzle sequences $\Pi_{t}^{\mathbf{S}}=\left\{P_{\left[s_{k}, t_{k}\right]}^{\mathbf{S}}\right\}_{k=1}^{\infty}$ and $\Pi_{v}^{\mathbf{S}}=\left\{P_{\left[u_{k}, v_{k}\right]}^{\mathbf{S}}\right\}_{k=1}^{\infty}$ whose limit contains $x$. Let $D_{r}(x)$ be a disc of radius $r>0$ centered at $x$. For every $k$, we can choose $r_{k}>0$ sufficiently small such that $D_{r_{k}}(x) \cap \mathcal{P}_{k}^{\mathbf{S}}=D_{r_{k}}(x) \cap\left(\overline{S_{1}} \cup \overline{S_{2}}\right)$. Let $N_{k} \geq 0$ be large enough such that $\left\{x_{i}\right\}_{i=N_{k}}^{\infty}$ is contained in $D_{r_{k}}(x)$. This implies that $\left\{x_{i}\right\}_{i=N_{k}}^{\infty} \subset P_{\left[s_{k}, t_{k}\right]}^{\mathbf{S}} \cup$ $P_{\left[u_{k}, v_{k}\right]}^{\mathbf{S}}$. It is easy to see that the sequence of image points $\left\{y_{i}=\Phi_{\mathbf{S}}\left(x_{i}\right)\right\}_{i=N_{k}}^{\infty}$ must be contained $P_{\left[s_{k}, t_{k}\right]} \cup P_{\left[u_{k}, v_{k}\right]}$. By Proposition $9.2, \Pi_{t}=\left\{P_{\left[s_{k}, t_{k}\right]}\right\}_{k=1}^{\infty}$ and $\Pi_{v}=\left\{P_{\left[u_{k}, v_{k}\right]}\right\}_{k=1}^{\infty}$ both converge to $y$, and the result follows.

Case ii) The proof is very similar to Case i), and hence, it will be omitted.

Case iii) Since $x$ is an iterated preimage of $\mathbf{k}_{\mathbf{S}}$, it must be the landing point of some bubble ray $\mathcal{R}_{t}^{\mathbf{S}}$, where $t \in \mathbb{R} / \mathbb{Z}$ is a dyadic rational. By Proposition 6.18, $y$ is the landing point of the corresponding bubble ray $\mathcal{R}_{t}^{\mathcal{S}}$. Any subsequence of $\left\{x_{i}\right\}_{i=0}^{\infty}$ contained in $\mathcal{R}_{t}^{\mathbf{S}}$ is mapped under $\Phi_{\mathbf{S}}$ to a sequence in $\mathcal{R}_{t}^{\mathcal{S}}$ which converges to $y$. Hence, we may assume that $x_{i}$ is not contained $\mathcal{R}_{t}^{\mathbf{S}}$ for all $i \geq 0$.

The remainder of the proof is very similar to Case $i)$, and hence, it will be omitted.

Case iv) By Proposition 6.11, there exists a unique maximal nested puzzle sequences $\Pi_{t}^{\mathbf{S}}=\left\{P_{\left[s_{k}, t_{k}\right]}^{\mathbf{S}}\right\}_{k=1}^{\infty}$ whose limit contains $x$. Let $D_{r}(x)$ be a disc of radius $r>0$ centered at $x$. Since $x$ is not contained the puzzle partition $\mathcal{P}_{n}^{\mathbf{S}}$ of any level $n \in \mathbb{N}$, it follows that for every $k \geq 1$, there exists $r_{k}>0$ sufficiently small such that $D_{r}(x) \subset P_{\left[s_{k}, t_{k}\right]}^{\mathrm{S}}$. Thus, there exists $N_{k} \geq 0$ such that $\left\{x_{i}\right\}_{i=N_{k}}^{\infty}$ is contained in 
$P_{\left[s_{k}, t_{k}\right]}^{\mathbf{S}}$. It is easy to see that the sequence of image points $\left\{y_{i}=\Phi_{\mathbf{S}}\left(x_{i}\right)\right\}_{i=N_{k}}^{\infty}$ must be contained in the corresponding puzzle piece $P_{\left[s_{k}, t_{k}\right]}$ for $R_{\nu}$. Since the nested puzzle sequence $\Pi_{t}=\left\{P_{\left[s_{k}, t_{k}\right]}\right\}_{k=1}^{\infty}$ must shrink to $y$, the result follows.

Proposition 9.4. Let $t \in \mathbb{R} / \mathbb{Z}$, and let $x \in J_{\mathbf{B}}$ and $y \in J_{\mathbf{S}}$ be the landing point of the external ray for $f_{\mathbf{B}}$ and $f_{\mathbf{S}}$ with external angle $-t$ and $t$ respectively. Then $\Phi_{\mathbf{B}}(x)=\Phi_{\mathbf{S}}(y)$.

Proof. Consider the nested puzzle sequences $\Pi_{t}^{\mathbf{B}}=\left\{P_{\left[s_{k}, t_{k}\right]}^{\mathbf{B}}\right\}_{k=1}^{\infty}, \Pi_{t}^{\mathbf{S}}=\left\{P_{\left[s_{k}, t_{k}\right]}^{\mathbf{S}}\right\}_{k=1}^{\infty}$ and $\Pi_{t}=\left\{P_{\left[s_{k}, t_{k}\right]}\right\}_{k=1}^{\infty}$. By Propositions 6.4 and 6.10, we have $L\left(\Pi_{t}^{\mathbf{B}}\right) \cap J_{\mathbf{B}}=\{x\}$ and $L\left(\Pi_{t}^{\mathbf{S}}\right) \cap J_{\mathbf{S}}=\{y\}$. Let $z$ be the point that $\Pi_{t}$ shrinks to. By definition, $\Phi_{\mathbf{B}}(x)=z=\Phi_{\mathbf{S}}(y)$.

Proof of Theorem B. We verify the conditions in Proposition 1.1. Let $f_{c_{1}}=f_{\mathbf{B}}$, $f_{c_{2}}=f_{\mathbf{S}}, \Lambda_{1}=\Phi_{\mathbf{B}}, \Lambda_{2}=\Phi_{\mathbf{S}}$, and $R=R_{\nu}$. Clearly, conditions (ii) and (iii) are satisfied. It remains to check condition (i).

Let $\tau_{\mathbf{B}}: \mathbb{R} / \mathbb{Z} \rightarrow J_{\mathbf{B}}$ and $\tau_{\mathbf{S}}: \mathbb{R} / \mathbb{Z} \rightarrow J_{\mathbf{S}}$ be the Carathéodory loop for $f_{\mathbf{B}}$ and $f_{\mathbf{S}}$ respectively (refer to Section 1 for the definition of Carathéodory loop). Define $\sigma_{\mathbf{B}}(t):=\tau_{\mathbf{B}}(-t)$. By Proposition 9.4 , the following diagram commutes:

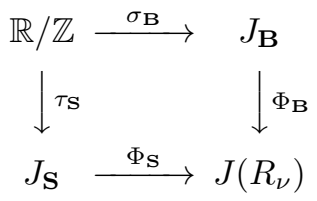

It follows that if $z \sim_{\text {ray }} w$, then $z$ and $w$ are mapped to the same point under $\Phi_{\mathbf{B}}$ or $\Phi_{\mathbf{S}}$.

To check the converse, it suffices to prove that for $z, w \in J_{\mathbf{S}}$, if $\Phi_{\mathbf{S}}(z)=\Phi_{\mathbf{S}}(w)=$ $x \in J\left(R_{\nu}\right)$, then $z \sim_{\text {ray }} w$. First, observe that $\Phi_{\mathbf{S}}$ maps iterated preimages of 0 homeomorphically onto the iterated preimages of 1 . Similarly, $\Phi_{\mathbf{S}}$ maps iterated preimages of $\mathbf{k}_{\mathbf{S}}$ homeomorphically onto the iterated preimages of $\kappa$. Now, by Proposition 6.20, two distinct maximal nested sequences for $R_{\nu}$ shrink to $x$ if and only if $x$ is an iterated preimage of $1, \kappa$ or $\beta$. If $x$ is an iterated preimage of 1 or $\kappa$, then $z$ must be equal to $w$. If $x$ is an iterated preimage of $\beta$, then $z \sim_{\text {ray }} w$.

\section{ACKNOWLEDGMEnTs}

The author would like to thank his advisor, Michael Yampolsky, for many stimulating discussions we had as this paper developed. The author is also grateful to the anonymous referee for the helpful comments.

\section{REFERENCES}

[AY] M. Aspenberg and M. Yampolsky, Mating non-renormalizable quadratic polynomials, Comm. Math. Phys. 287 (2009), no. 1, 1-40, DOI 10.1007/s00220-008-0598-y. MR2480740 (2009k:37098)

[BF] B. Branner, N. Fagella, Quasiconformal Surgery in Holomorphic Dynamics, Cam. Stud. in Adv. Math. (2014).

[Do] A. Douady, Systèmes dynamiques holomorphes (French), Bourbaki seminar, Vol. 1982/83, Astérisque, vol. 105, Soc. Math. France, Paris, 1983, pp. 39-63. MR728980 (85h:58090)

[DH] A. Douady and J. H. Hubbard, Étude dynamique des polynômes complexes I and II, Pub. math. d'Orsay 84-02 et 85-05, (1984/1985).

[Du] D. Dudko, Mating with Laminations, arXiv:1112.4780. 
[Ha] P. Haïssinsky, Chirurgie parabolique, C. R. Acad. Sci. Paris Sér. I Math. 327 (1998), no. 2, 195-198, DOI 10.1016/S0764-4442(98)80088-8. MR.1645124 (99i:58127)

[Lu] J. Luo, Combinatorics and holomorphic dynamics: Captures, matings, Newton's method, ProQuest LLC, Ann Arbor, MI, 1995. Thesis (Ph.D.)-Cornell University. MR2691795

[M1] J. Milnor, Dynamics in one complex variable, 3rd ed., Annals of Mathematics Studies, vol. 160, Princeton University Press, Princeton, NJ, 2006. MR.2193309 (2006g:37070)

[M2] J. Milnor, Periodic orbits, externals rays and the Mandelbrot set: an expository account, Astérisque 261 (2000), xiii, 277-333. Géométrie complexe et systèmes dynamiques (Orsay, 1995). MR1755445 (2002e:37067)

[Mc] C. T. McMullen, Complex dynamics and renormalization, Annals of Mathematics Studies, vol. 135, Princeton University Press, Princeton, NJ, 1994. MR.1312365 (96b:58097)

[P] C. L. Petersen, Local connectivity of some Julia sets containing a circle with an irrational rotation, Acta Math. 177 (1996), no. 2, 163-224, DOI 10.1007/BF02392621. MR.1440932 (98h:58164)

[Re] M. Rees, Realization of matings of polynomials as rational maps of degree two, Manuscript, (1986).

[Tan] L. Tan, Matings of quadratic polynomials, Ergodic Theory Dynam. Systems 12 (1992), no. 3, 589-620, DOI 10.1017/S0143385700006957. MR.1182664 (93h:58129)

[S] C. L. Siegel, Iteration of analytic functions, Ann. of Math. (2) 43 (1942), 607-612. MR.0007044(4,76c)

[Shi] M. Shishikura, On a theorem of M. Rees for matings of polynomials, The Mandelbrot set, theme and variations, London Math. Soc. Lecture Note Ser., vol. 274, Cambridge Univ. Press, Cambridge, 2000, pp. 289-305. MR1765095 (2002d:37072)

[Sw] G. Świątek, Rational rotation numbers for maps of the circle, Comm. Math. Phys. 119 (1988), no. 1, 109-128. MR968483 (90h:58077)

[Y1] M. Yampolsky, Complex bounds for renormalization of critical circle maps, Ergodic Theory Dynam. Systems 19 (1999), no. 1, 227-257, DOI 10.1017/S0143385799120947. MR1677153 (2000d:37053)

[Y2] M. Yampolsky, Siegel disks and renormalization fixed points, Holomorphic dynamics and renormalization, Fields Inst. Commun., vol. 53, Amer. Math. Soc., Providence, RI, 2008, pp. 377-393. MR2477430 (2009k:37107)

[YZ] M. Yampolsky and S. Zakeri, Mating Siegel quadratic polynomials, J. Amer. Math. Soc. 14 (2001), no. 1, 25-78 (electronic), DOI 10.1090/S0894-0347-00-00348-9. MR.1800348 (2001k:37064)

Department of Mathematics, University of Toronto, 100 St. George St., Toronto ON M5S 3G3, CANADA

E-mail address: jonguk.yang@mail.utoronto.ca 